\title{
A Quasiphysical and Dynamic Adjustment Approach for Packing the Orthogonal Unequal Rectangles in a Circle with a Mass Balance: Satellite Payload Packing
}

\author{
Ziqiang Li, ${ }^{1}$ Xianfeng Wang, ${ }^{1}$ Jiyang Tan, ${ }^{2}$ and Yishou Wang ${ }^{3}$ \\ ${ }^{1}$ School of Information and Engineering, Xiangtan University, Xiangtan, Hunan 411105, China \\ ${ }^{2}$ School of Mathematics and Computer Science, Xiangtan University, Xiangtan, Hunan 411105, China \\ ${ }^{3}$ Department of Aeronautics, Xiamen University, Xiamen, Fujian 361005, China \\ Correspondence should be addressed to Ziqiang Li; xtulzq@sina.com
}

Received 2 June 2014; Revised 28 August 2014; Accepted 22 September 2014; Published 2 December 2014

Academic Editor: Oleg V. Gendelman

Copyright (C) 2014 Ziqiang Li et al. This is an open access article distributed under the Creative Commons Attribution License, which permits unrestricted use, distribution, and reproduction in any medium, provided the original work is properly cited.

\begin{abstract}
Packing orthogonal unequal rectangles in a circle with a mass balance (BCOURP) is a typical combinational optimization problem with the NP-hard nature. This paper proposes an effective quasiphysical and dynamic adjustment approach (QPDAA). Two embedded degree functions between two orthogonal rectangles and between an orthogonal rectangle and the container are defined, respectively, and the extruded potential energy function and extruded resultant force formula are constructed based on them. By an elimination of the extruded resultant force, the dynamic rectangle adjustment, and an iteration of the translation, the potential energy and static imbalance of the system can be quickly decreased to minima. The continuity and monotony of two embedded degree functions are proved to ensure the compactness of the optimal solution. Numerical experiments show that the proposed QPDAA is superior to existing approaches in performance.
\end{abstract}

\section{Introduction}

$2 \mathrm{D}$ rectangle packing problems are derived from the industry and antiaircraft field [1-3]. They occur in logistics packing, plate cutting, the layout design of the very large scale integration (VLSI), and satellite modules. They can be divided into unconstrained rectangle packing problems [1] and constrained ones [3]. Both are NP-hard problems and are difficult to be solved. However they have attracted much attention and some packing approaches for different containers have been reported in literatures.

For the $2 \mathrm{D}$ rectangle container, the packing approaches mainly include graph theories [4-7], branch-and-bound methods [8-10], dynamic planning [11], heuristics [12-15], artificial intelligent [16], evolutionary approaches [17], and hybrid approaches [18-20]. Regarding the strip container, main packing approaches are branch-and-bound methods [21], heuristics [22, 23], and evolutionary approaches [24, 25]. On the $2 \mathrm{D}$ polygon or $3 \mathrm{D}$ polyhedron container, the existing packing approaches have heuristics [26], evolutionary approaches [27-29], and integer programming [30]. Some scholars are interested in the packing problem of the convex region and have proposed heuristics [26] and branch-andbound approaches [31].

The layout design problem of the satellite module described in [32] is an important packing problem, which can be transformed into the problem of packing 2D orthogonal unequal rectangles within a circular container with the mass balance (BCOURP). In 1999, Feng et al. [33] built a mathematical model of this problem and analyzed the isomorphism and equivalent intrinsic properties among its layout schemes by using the graph theory and group theory and proposed a theoretical global optimization algorithm. In 2007, Xu et al. [34] defined embedded degree functions between two rectangles and between the rectangle and circular container and presented a compaction algorithm with the particle swarm local search (CA-PSLS). Their idea is that a feasible solution with a smaller envelope radius obtained through the gradient method is taken as an elite individual and the optimal solution is obtained by the PSO iteration. In 2010, Xu 


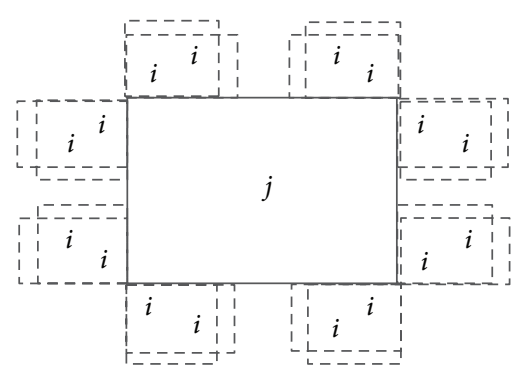

FIGURE 1: The sketch map for available positions of the rectangle.

et al. [35] suggested a heuristic algorithm ordered by GA (GAHA, see its algorithm steps in Appendix A and Figure 1). Its key technology is the positioning strategy of constructing the feasible solution. By combining it with GA, the computational efficiency and solution quality are improved.

Generally, there exist strong points and deficiencies for each type of approaches.

(i) For approaches based on the graph theory, there exists combinatorial explosion when the adjacent topological relation is transformed into the layout diagraph without a size limit for the large-scale layout problem. This is because only two limited relations called the vicinity and distance can be used in pruning branch.

(ii) The heuristic method can be used to quickly construct a feasible solution. But it is generally not easy to devise a good heuristic strategy, unless the designer makes a long time painstaking effort and has good luck.

(iii) Stochastic algorithms have the global search ability, but there exists the bottleneck of time-costing overlapping area calculation for them [33]. By combining the heuristic method with the stochastic algorithm, their respective advantages can be exerted to the utmost. Based on this mechanism, CA-PSLS and GA$\mathrm{HA}$ are consecutively proposed to solve this problem. According to No Free Lunch Theory [36], how to obtain the knowledge from the problem itself and its area and fuse it into the heuristic and stochastic search mechanism is a way of designing a high performance approach for this problem.

Huang et al. [37-40] presented a quasiphysical and quasihuman heuristic algorithm and its variants for the circle packing problem. They obtained excellent results. For BCOURP, Xu et al. [34] proposed CA-PSLS based on embedded degrees between circumcircles of two rectangles and between the container and rectangle's circumcircle. But due to the discontinuity of the two embedded degree functions, it is difficult to obtain a high quality solution by using CA-PSLS. That is, constructing the continuous rectangular embedded function and exploring a better optimized mechanism are necessary for this problem. Therefore, in this paper, we consider two definitions of monotonous and continuous embedded degrees between two orthogonal rectangles and between an orthogonal rectangle and the container and suggest a dynamic adjustment strategy. We merge them into the proposed QPDAA to improve the solution quality of BCOURP. Numerical experiments will test effectiveness of the considered QPDAA.

The remainder of this paper is organized as follows. The problem statement and mathematical model are in Section 2. The compact and feasible solution strategy and dynamic adjustment strategy are given in Sections 3 and 4, respectively. This algorithm is presented in Section 5. Section 6 is experiments and analysis. The conclusion is shown in Section 7. The final part is acknowledgment.

\section{Problem Statement and Mathematical Model}

Consider the following two related definitions where $\mathbf{I}_{n}=$ $\{1,2, \ldots, n\}$ and $n$ is the number of rectangles.

Definition 1. As shown in Figure 2, let the origin of the Cartesian coordinate system be the center of the container. Let $R_{i}$ denote the $i$ th $(i=1,2, \ldots, n)$ rectangle and let $\left(x_{i}, y_{i}\right), l_{i}, w_{i}, m_{i}$, and $\theta_{i}$ be its center, length, width, mass, and direction angle between its long side and the positive direction of the $x$-axis, respectively. Then a layout scheme of $n$ rectangles $R_{i}\left(x_{i}, y_{i}, l_{i}, w_{i}, m_{i}, \theta_{i}\right)(i=1,2, \ldots, n)$ can be denoted by $\mathbf{X}^{\prime}\left(x_{i}, y_{i}, \theta_{i} \mid i=1,2, \ldots, n\right)$.

Definition 2. For a layout scheme $\mathbf{X}^{\prime}$, if $\theta_{i}=0^{\circ}$ or $90^{\circ}$, then $\mathbf{X}^{\prime}$ is an orthogonal rectangle packing scheme, $R_{i}\left(i \in \mathbf{I}_{n}\right)$ is an orthogonal rectangle, and the packing is the orthogonal rectangle packing (see Figure 3 ).

Herein this paper considers only orthogonal rectangle packing schemes.

Suppose that the center of each rectangle coincides with its mass center. Let $T$ be a rectangle set $\left\{R_{1}, R_{2}, \ldots, R_{n}\right\}$; then the mathematical model of this problem can be described as follows. Find a solution $\mathbf{X}=\left(x_{i}, y_{i}, \theta_{i} \mid i=1,2, \ldots, n\right)^{T}$ and $\mathbf{X}$ satisfies Formulas (1)-(4). Consider

$$
\begin{array}{ll}
\min & f(\mathbf{X}) \\
\text { s.t. } & \operatorname{int}\left(R_{i}\right) \cap \operatorname{int}\left(R_{j}\right)=\emptyset, \quad i, j=1,2, \ldots, n, i \neq j \\
& \operatorname{int}\left(R_{i}\right) \cap \operatorname{int}\left(R_{c}\right)=\operatorname{int}\left(R_{i}\right) \quad i=1,2, \ldots, n \\
& J(X)=\sqrt{\left(\sum_{i=1}^{n} m_{i} x_{i}\right)^{2}+\left(\sum_{i=1}^{n} m_{i} y_{i}\right)^{2}} \leq \delta .
\end{array}
$$




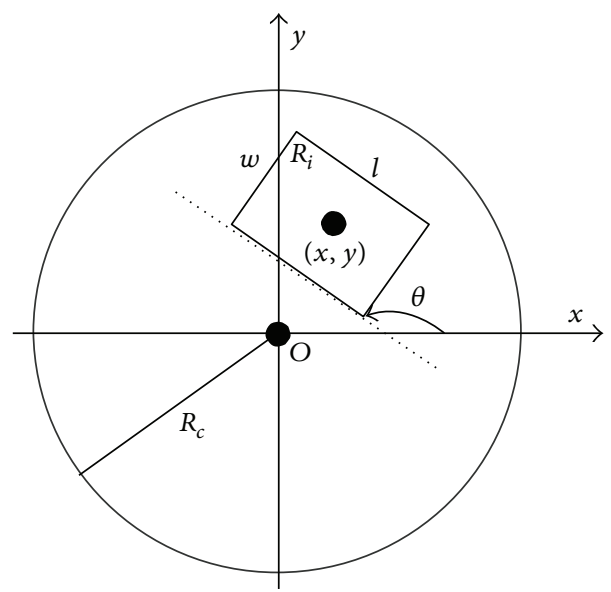

FIGURE 2: The definition of a rectangle.

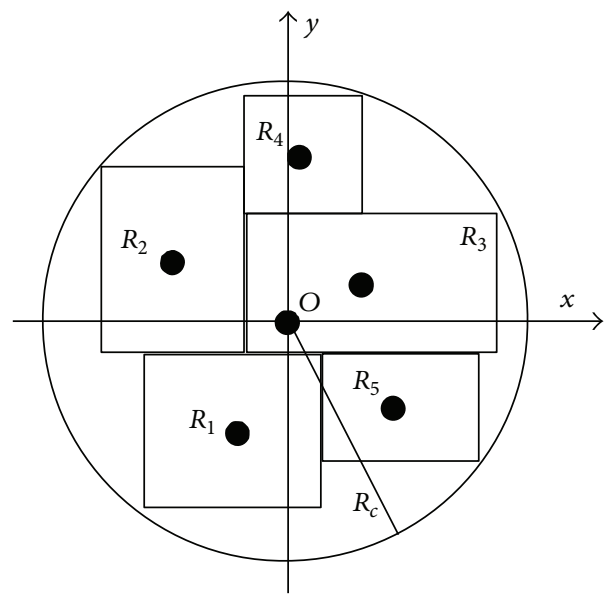

FIGURE 3: The orthogonal rectangle packing scheme.

In Formula (1), $f(\mathbf{X})$ denotes the radius of the enveloping circle of the scheme $\mathbf{X}$ whose circular center is at $(0,0)$. Formula (2) indicates that there is no overlap region between two rectangles $R_{i}$ and $R_{j}$. Formula (3) indicates that all rectangles are contained in the container. In Formulas (2) and (3), $\operatorname{int}\left(R_{i}\right)$ denotes the interior region of the rectangle $R_{i}$. Formula (4) means that the static imbalance $J(\mathbf{X})$ of the solution $\mathbf{X}$ is less than its threshold $\delta$, where $\delta>0$.

\section{Compact and Feasible Solution Strategy}

Based on the potential energy function of the embedded degree between two circles, Huang et al. [38-40] proposed the quasiphysical strategy and its variants for the circle packing problem. Inspired by the quasiphysical idea, we suggest a compact and feasible strategy for BCOURP.

3.1. Embedded Degree Function and Related Properties. Xu et al. [34] defined the embedded degrees between two rectangles and between the rectangle and container by Definitions 3 and 4 , respectively.
Definition 3. Let $r_{i}^{\prime}$ and $r_{j}^{\prime}(i, j=1,2, \ldots, n$ and $i \neq j)$ denote the radii of the circumscribed circles of two rectangles $R_{i}$ and $R_{j}$, respectively, and $d_{i j}$ (see Figure $4(\mathrm{a})$ ) the embedded degree between them (see Figure 4(a)); then $d_{i j}$ can be calculated by

$$
d_{i j}
$$

$$
= \begin{cases}r_{i}^{\prime}+r_{j}^{\prime} & \\ -\sqrt{\left(x_{i}-x_{j}\right)^{2}+\left(y_{i}-y_{j}\right)^{2}} & \text { if } R_{i} \text { and } R_{j} \text { overlap } \\ & (i, j=1,2, \ldots, n, i \neq j) \\ 0 & \text { otherwise. }\end{cases}
$$

Definition 4. Let $R_{c}$ and $r_{i}^{\prime}(i=1,2, \ldots, n)$ denote the radii of the container and circumscribed circle of the rectangle $R_{i}$, respectively, and $d_{0 i}$ (see Figure $4(\mathrm{~b})$ ) the embedded 


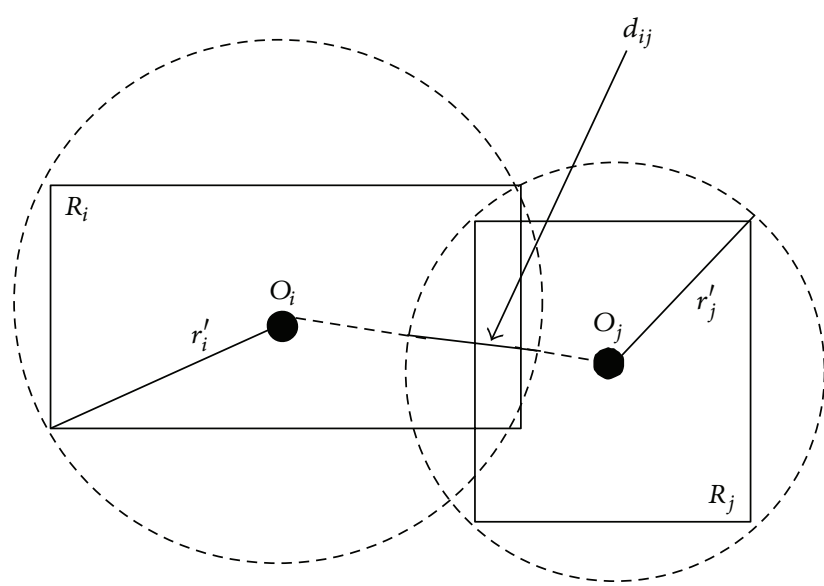

(a) The embedded degree between two rectangles

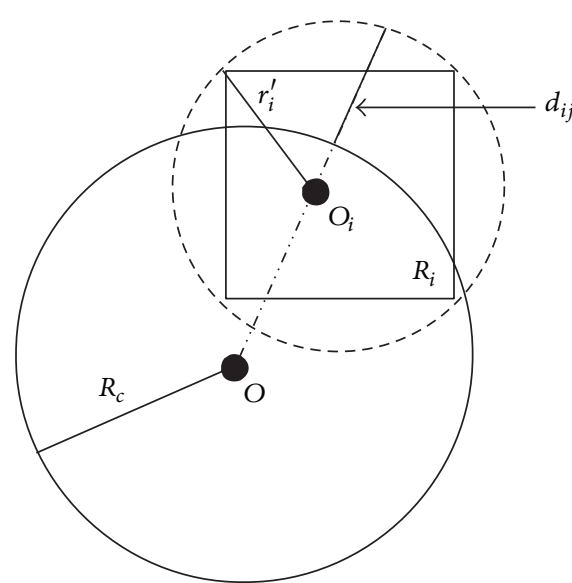

(b) The embedded degree between the rectangle and container

FIgure 4: Two embedded degree definitions in [34].

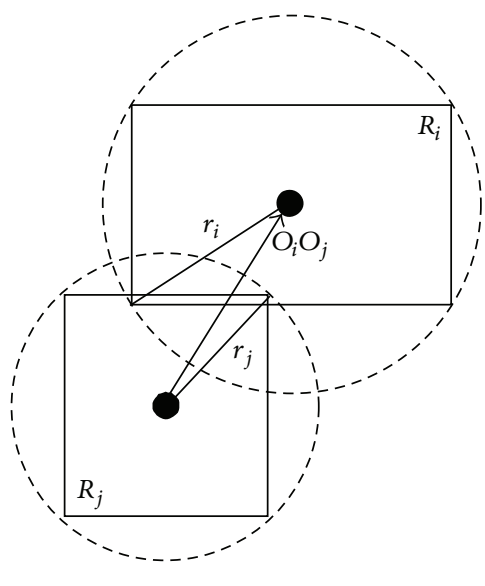

State 1

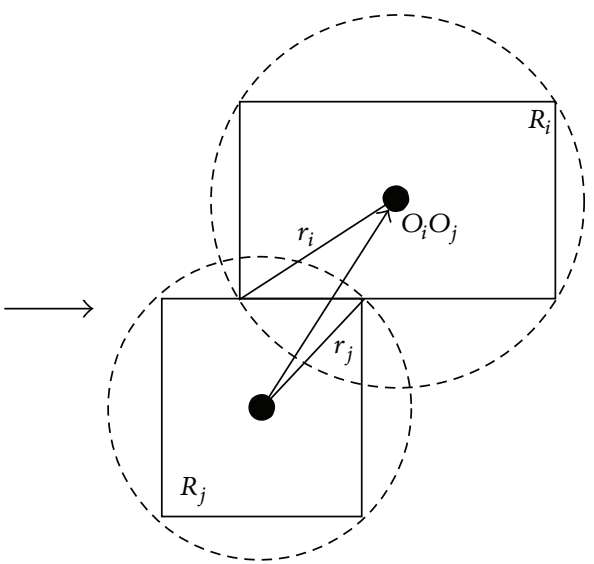

State 2

FIGURE 5: The embedded degree between two rectangles in the critical state.

degree between the rectangle and container; then $d_{0 i}$ can be calculated by

$$
\begin{aligned}
& d_{0 i} \\
& = \begin{cases}\sqrt{x_{i}^{2}+y_{i}^{2}}+r_{i}^{\prime}-R_{c} & \text { if } R_{i} \text { overlap with the container } \\
0 & \text { otherwise. }\end{cases}
\end{aligned}
$$

Both of the above two embedded degree functions are discontinuous in the critical state from overlapping to separating, as has been discussed by Stoyan and Yaskov [41]. For example, as shown in Figure 5, by moving one rectangle along a direction, two rectangles with the overlap area of $10^{-5}$ (state 1) are changed into $R_{i}\left(2.5,5,6,5,30,0^{\circ}\right)$ and $R_{j}(0,0,5,4$, $20,90^{\circ}$ ) (state 2). By Formula (5), we know that $d_{i j}=r_{i}+$ $r_{j}-O_{i} O_{j} \approx 1.52$ for state 1 , but $d_{i j}=0$ for state 2 . So, $d_{i j}$ in Definition 3 is discontinuous where $d_{i j}=0(i, j=1,2, \ldots, n$ and $i \neq j)$. Similarly, $d_{0 i}(i=1,2, \ldots, n)$ in Definition 4 is also discontinuous (see Figure 6). Owing to their discontinuity, it is difficult to select an appropriate step length to obtain the feasible and compact layout scheme for the gradient iteration of CA-PSLS. Inspired by [41], Definitions 5 and 6 are given for the considered QPDAA.

Definition 5. For two rectangles $R_{i}$ and $R_{j}\left(\theta_{i}, \theta_{j}=0^{\circ}\right.$ or $90^{\circ}$, $i, j=1,2, \ldots, n$ and $i \neq j$ ) (shown in Figure 7), let $r_{i}$ and $r_{j}$ be the radii of their circumscribed circles, respectively, and let $d_{i j}$ denote their embedded degree; then $d_{i j}$ can be calculated by

$$
\begin{aligned}
& d_{i j} \\
& =\left\{\begin{array}{lr}
\frac{\left(r_{i}+r_{j}\right)}{\left(\left(\log _{\left(a_{i}+a_{j}\right)}^{u}\right)^{2}+\left(\log _{\left(b_{i}+b_{j}\right)}^{v}\right)^{2}+1\right)}, & \text { If } 0<u \leq 1, \\
0 & 0<v \leq 1 \\
0 & \text { Otherwise. }
\end{array}\right.
\end{aligned}
$$




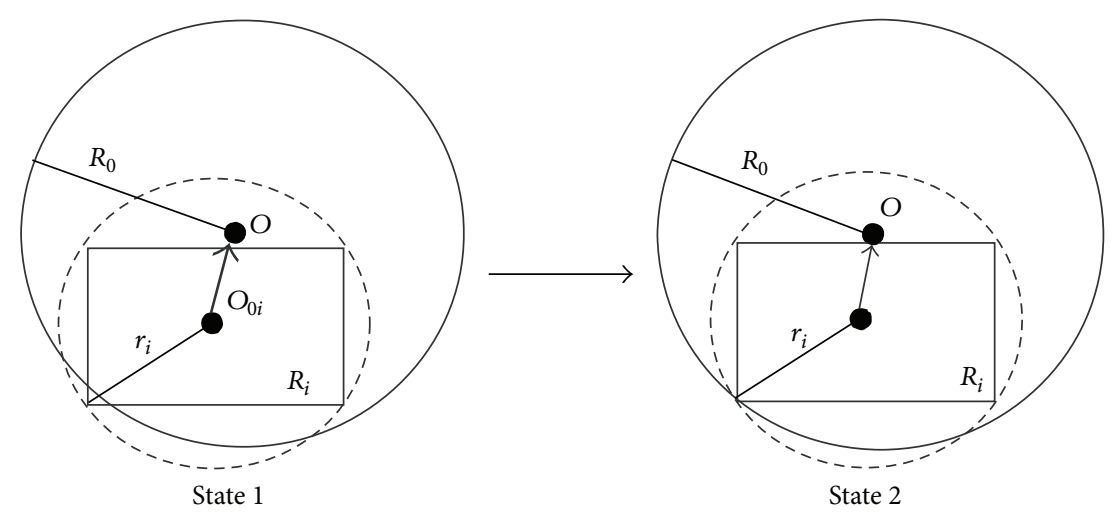

FIGURE 6: The embedded degree between the rectangle and container in the critical state.

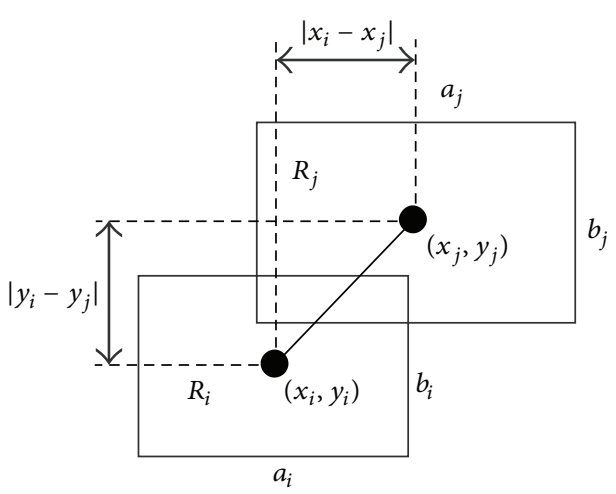

(a) $\left|\theta_{i}-\theta_{j}\right|=0$

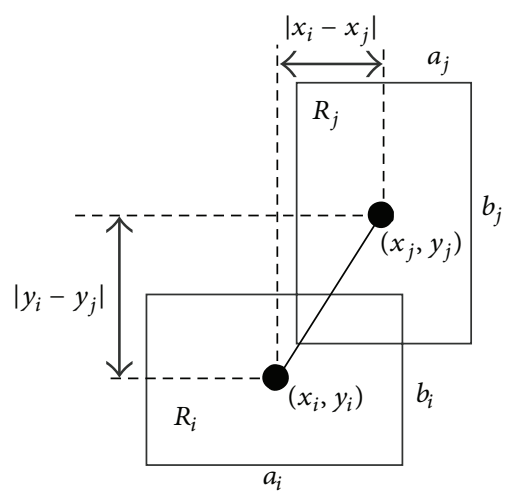

(b) $\left|\theta_{i}-\theta_{j}\right|>0$

FIGURE 7: Two cases of the embedded degree definition between two rectangles.

In Formula (7), $u=\left(a_{i}+a_{j}-2\left|x_{i}-x_{j}\right|\right) /\left(a_{i}+a_{j}\right), v=\left(b_{i}+b_{j}-\right.$ $\left.2\left|y_{i}-y_{j}\right|\right) /\left(b_{i}+b_{j}\right)$. Here, if $\theta_{i}=0^{\circ}, a_{i}=l_{i}, a_{j}=l_{j}, b_{i}=w_{i}$, and $b_{j}=w_{j}\left(\right.$ see Figure 7(a)); otherwise, $a_{i}=w_{i}, a_{j}=w_{j}, b_{i}=l_{i}$, and $b_{j}=l_{j}$ (see Figure 7(b)).

In Formula (7), the embedded degree between two rectangles is the moving distance of the rectangle $R_{j}$ from an overlap state with the stationary $R_{i}$ to the separation state along the direction from the center $\left(x_{i}, y_{i}\right)$ to the center $\left(x_{j}, y_{j}\right)$. If $R_{i}$ and $R_{j}$ are two squares, and the center of $R_{j}$ is on the diagonal line of $R_{i}$ and enough close to its center (i.e., $x_{i} \rightarrow x_{j}$ and $y_{i} \rightarrow y_{j}$ ), the moving distance of $R_{j}$ from the initial state (shown in Figure 8(a)) to the separate state (shown in Figure 8(b)) along the direction of their diagonal lines is about $r_{i}+r_{j}$. Thus in the initial state, their embedded degree $d_{i j}$ is close to the maximal value $r_{i}+r_{j}$. In addition, when $2\left|x_{i}-x_{j}\right| \rightarrow a_{i}+a_{j}$ and/or $2\left|y_{i}-y_{j}\right| \rightarrow b_{i}+b_{j}, u \rightarrow 0$ and/or $v \rightarrow 0$. That is, $d_{i j} \rightarrow 0$.

Definition 6. For the rectangle $R_{i}\left(\theta_{i}=0^{\circ}\right.$ or $90^{\circ}, i=$ $1,2, \ldots, n)$ and the circle container $\left(0,0, R_{c}\right)$ as shown in
Figure 7, let $d_{0 i}$ denote the embedded degree between the rectangle $R_{i}$ and container; then $d_{0 i}$ can be calculated by

$$
d_{0 i}=\left\{\begin{array}{c}
\sqrt{\left(\left|x_{i}\right|+\frac{l_{i}}{2}\right)^{2}+\left(\left|y_{i}\right|+\frac{w_{i}}{2}\right)^{2}}-R_{c} \\
0 \sqrt{\left(\left|x_{i}\right|+\frac{l_{i}}{2}\right)^{2}+\left(\left|y_{i}\right|+\frac{w_{i}}{2}\right)^{2}}>R_{c} \\
\sqrt{\left(\left|x_{i}\right|+\frac{l_{i}}{2}\right)^{2}+\left(\left|y_{i}\right|+\frac{w_{i}}{2}\right)^{2}} \leq R_{c} \\
(i=1,2, \ldots, n) .
\end{array}\right.
$$

The geometric interpretation of Definition 6 is that when the farthest vertex of the rectangle $R_{i}(i=1,2, \ldots, n)$ from the coordinate origin is within the container, their embedded degree $d_{0 i}=0$; otherwise it is the length of the straight line segment pointed by $d_{0 i}$ in Figure 9.

For embedded degree functions in Definitions 3 and 5, their geometric figures are two curved semi-cone surfaces 


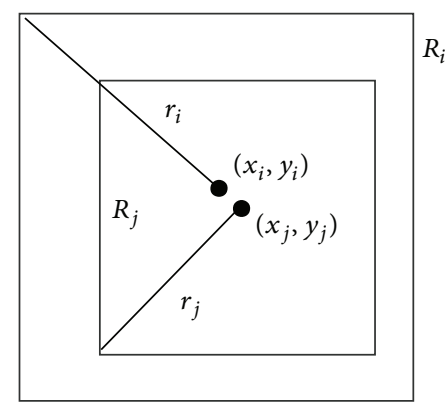

(a) Initial state

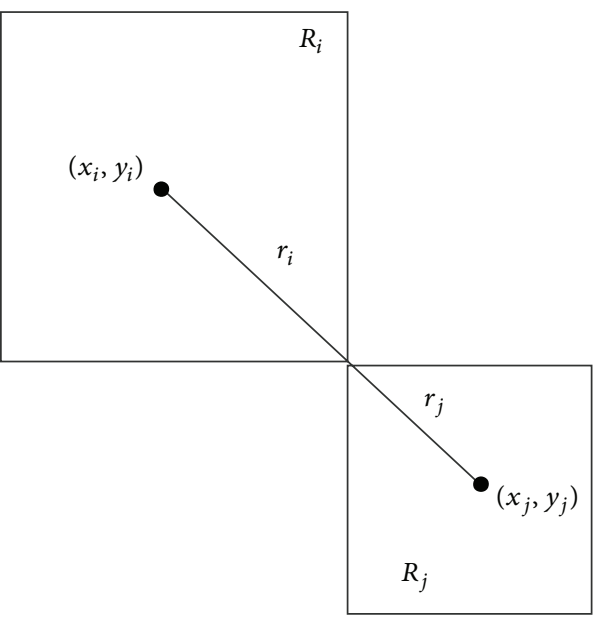

(b) Separation state

FIGURE 8: The geometric interpretation of definition of the embedded degree between two rectangles.

shown in Figures 10(a) and 10(b), respectively, where it is obvious that there is a gap between the semi-cone surface and xoy plane in Figure 10(a) but there is no gap between them in Figure 10(b). It is not difficult to assert that the difference of geometric figures of two embedded degree functions in Definitions 4 and 6 is the same as the above one. After describing Lemma 7, we propose properties of two embedded degree functions in Definitions 5 and 6, respectively.

Lemma 7. If a binary function $f(x, y)$ is continuous for each variable in a domain, respectively, and is monotonous for the variable $x$ or $y$, then the function $f(x, y)$ is continuous in the domain.

Property1. $\forall i, j \in \mathbf{I}_{n}$, let $R_{i}$ and $R_{j}\left(i \neq j\right.$ and $\theta_{i}, \theta_{j}=0^{\circ}$ or $\left.90^{\circ}\right)$ be two rectangles, and the domain $\mathbf{D}=\{u \leq 1$ and $v \leq 1\}$. Then $d_{i j}(u, v)$ in Definition 5 is a continuous binary function in the domain $\mathbf{D}$.

Proof. $\forall i, j \in \mathbf{I}_{n}$ and $i \neq j$, set $\mathbf{D}_{1}=\{(u, v) \mid 0<u \leq$ 1 and $0<v \leq 1\}$. From Definition 5, we know that the function $d_{i j}(u, v)$ is continuous on both $\mathbf{D}_{1}$ and $\mathbf{D}-\mathbf{D}_{1}$. Here, we prove that it is continuous on the domain $\mathbf{D}_{2}\{(u, v)$ | $(u, v) \in \mathbf{D}$ and $u=0$ or $v=0\}$.

For $(u, v) \in \mathbf{D}_{1}$,

$$
\lim _{u \rightarrow 0} d_{i j}(u, v)=\lim _{u \rightarrow 0} \frac{r_{i}+r_{j}}{\left(\log _{a_{i}+a_{j}}^{u}\right)^{2}+\left(\log _{b_{i}+b_{j}}^{v}\right)^{2}+1}=0 .
$$

Simultaneously,

$$
\lim _{v \rightarrow 0} d_{i j}(u, v)=\lim _{u \rightarrow 0, v \rightarrow 0} d_{i j}(u, v)=0 \quad \text { for }(u, v) \in \mathbf{D}_{1} .
$$

This is because

$$
\begin{array}{r}
\frac{\partial d_{i j}(u, v)}{\partial u} \\
=\frac{-\left(r_{i}+r_{j}\right)\left(a_{i}+a_{j}\right) \log _{a_{i}+a_{j}}^{u}}{u \ln \left(a_{i}+a_{j}\right)\left(\left(\log _{a_{i}+a_{j}}^{u}\right)^{2}+\left(\log _{b_{i}+b_{j}}^{v}\right)^{2}+1\right)^{2}}>0 \\
\text { for }(u, v) \in \mathbf{D}_{1} .
\end{array}
$$

According to Lemma 7, the binary function $d_{i j}(u, v)$ is continuous on the domain $\mathbf{D}_{2}$. Therefore, $d_{i j}(u, v)$ is continuous on the domain $\mathbf{D}$.

Property 2. $\forall i, j \in \mathbf{I}_{\mathbf{n}}$ and $i \neq j$, for the container with the radius $R_{c}$ and rectangle $R_{i}\left(\theta_{i}=0^{\circ}\right.$ or $\left.90^{\circ}, i=1,2, \ldots, n\right)$, set $u=x_{i}$ and $v=y_{i}$; then the binary function $d_{0 i}$ in Definition 6 is continuous on the domain $\mathbf{D}_{3}\{(u, v) \mid-\infty<u<$ $+\infty$ and $-\infty<v<+\infty\}$.

3.2. Extruded Force and Energy Function. In order to quickly decrease the overlapping area of the rectangle packing system, we define the extruded forces between two rectangles and between the rectangle and container.

Definition 8. Let $R_{i}$ and $R_{j}$ be two rectangles with the embedded degree $d_{i j}(i, j=1,2, \ldots, n$ and $i \neq j)$. Then the extruded force $\overrightarrow{F_{j i}}$ between $R_{i}$ and $R_{j}$ is calculated by

$$
\overrightarrow{F_{j i}}=\frac{\alpha d_{j i} \overrightarrow{d_{j i}}}{\left|\overrightarrow{d_{j i}}\right|} \quad(i, j=1,2, \ldots, n, i \neq j) .
$$

Definition 9. Let $R_{i}(i=1,2, \ldots, n)$ and $R_{c}$ denote the rectangle $i$ and container. Then the extruded force $\overrightarrow{F_{0 i}}$ between $R_{i}$ and $R_{c}$ can be calculated by Formula (13), whose direction 


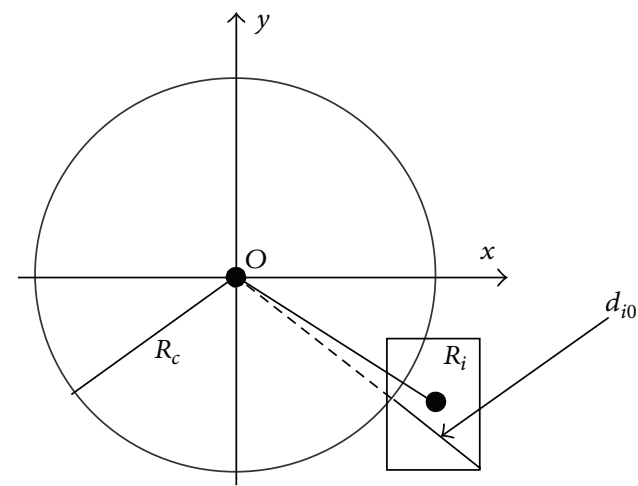

FiguRE 9: The schematic diagram overlapped between the orthogonal rectangle and container.

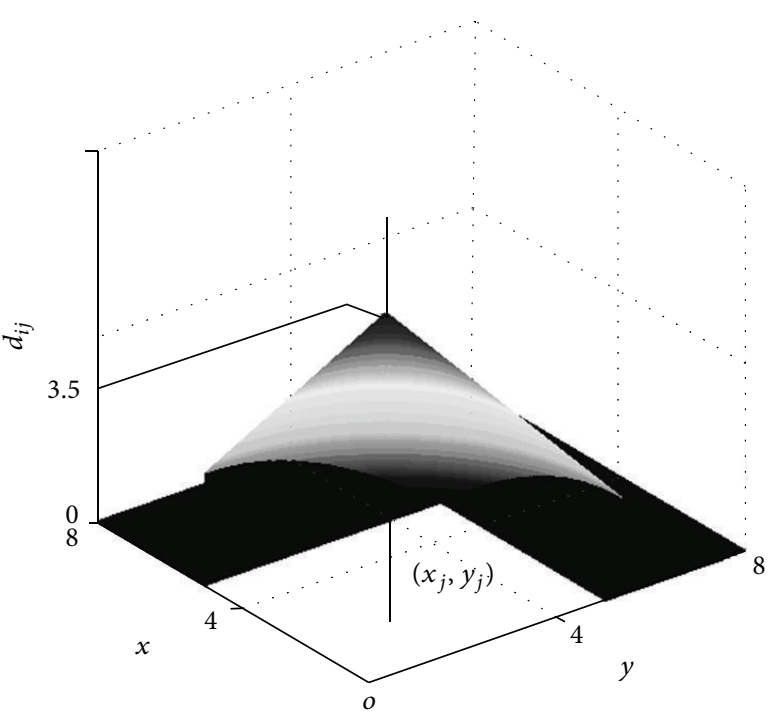

(a) Definition 3

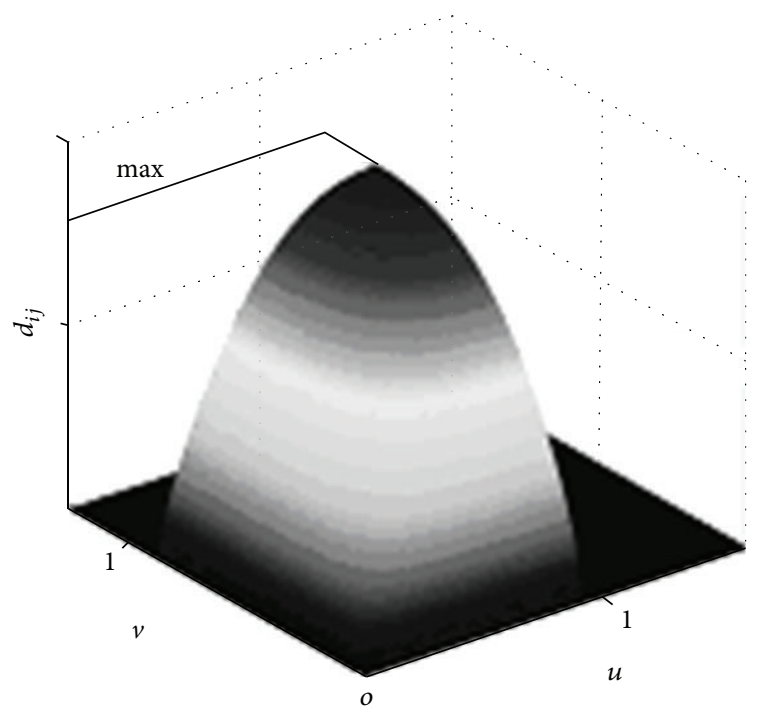

(b) Definition 5

FIGURE 10: The curved semi-cone surface of the embedded degree of two orthogonal rectangles.

is the direction from the center of the container to the furthermost rectangular vertex (see Figure 6):

$$
\overrightarrow{F_{0 i}}=\frac{\beta d_{0 i} \overrightarrow{d_{0 i}}}{\left|\overrightarrow{d_{0 i}}\right|} \quad(i=1,2, \ldots, n)
$$

By experiments, we can know that $\alpha>\beta$.

So, the extruded resultant force $\vec{F}_{i}$ of $R_{i}(i=1,2, \ldots, n)$ in the rectangle packing scheme can be calculated by

$$
\vec{F}_{i}=\sum_{j=0, i \neq j}^{n} \overrightarrow{F_{j i}}
$$

Definition 10. Let $E_{j i}$ and $E_{0 i}(i, j=1,2, \ldots, n$ and $i \neq j)$ denote extruded potential energies of $R_{i}$ with respect to $R_{j}$ and the container, respectively. Then $E_{j i}$ can be calculated by Formula (15), where $d_{i j}$ and $d_{i 0}$ denote two embedded degrees between two rectangles $R_{i}$ and $R_{j}$ and between the rectangles $R_{i}$ and container:

$$
E_{j i}=d_{j i}^{2} \quad(i=1,2, \ldots, n, j=0,1, \ldots, n, i \neq j) .
$$

Definition 11. The total extruded potential energy $E_{i}$ of $R_{i}$ can be calculated by

$$
E_{i}=\sum_{j=0, j \neq i}^{n} E_{j i} \quad(i, j=1,2, \ldots, n, i \neq j) .
$$

Let $S_{i}$ be the area of the rectangle $R_{i}$; then $E_{i}$ and $E_{i} / S_{i}$ $(i=1,2, \ldots, n)$ are its absolute and relative extruded potential energies, respectively.

3.3. Compact and Feasible Solution Strategy. By predetermining envelope radius $R_{0}$ of this problem, the extruded force and direction can be calculated by Formula (13). The extruded force of the envelope circle makes each rectangle close to the center of the container in the direction. For each rectangle, 
TABle 1: Parameters of rectangles for five layout examples.

\begin{tabular}{|c|c|}
\hline Example 1 & (length, width, mass) $(8,6,12),(8,8,16),(10,6,15),(12,4,12),(6,6,9)$ \\
\hline Example 2 & (length, width, mass $)(8,6,12),(8,8,16),(10,6,15),(10,8,20),(10,10,25),(12,6,18)$ \\
\hline Example 3 & (length, width, mass) $(8,6,12),(8,8,16),(10,6,15),(10,8,20),(10,10,25),(12,6,18),(12,4,12),(12,8,24)(12,10,30)$ \\
\hline Example 4 & $\begin{array}{l}\text { (length, width, mass })(8,5,10),(4,8,8),(10,6,15),(7,8,14),(10,3,7.5),(12,6,18),(12,4,12),(12,6,18),(8,10,20),(7,3,6) \text {, } \\
(8,6,6),(8,3,15),(10,6,20),(10,8,17.5),(10,7,15),(12,5,15),(12,4,12),(10,8,20),(12,10,30),(6,6,9)\end{array}$ \\
\hline Example 5 & $\begin{array}{l}\text { (length, width, mass })(26,26,8),(22,28,16),(20,30,16),(20,34,15),(34,22,17),(20,32,15),(30,20,14),(28,26,15), \\
(20,28,14),(26,38,13),(34,30,12),(26,36,17),(30,34,12),(28,24,17)(32,24,10),(30,38,11),(30,20,18),(22,22,17), \\
(30,32,11),(28,28,10),(20,38,18),(22,30,13)(32,32,14),(26,26,17),(34,20,14),(26,32,18),(38,22,19),(36,34,18), \\
(36,24,13),(26,30,15)(26,38,12),(30,38,19),(28,24,15),(38,34,19),(26,20,14),(32,36,16),(28,22,13),(24,28,19), \\
(38,22,17),(20,38,16)\end{array}$ \\
\hline
\end{tabular}

the extruded forces of all others with respect to the rectangle drive away themselves to relieve the pressure in respective direction and their extruded resultant force is calculated by Formula (12). In this paper, the above two steps are used to decrease the overlapping area of the packing scheme and make it compact.

\section{Dynamic Adjustment Strategy}

Considering the problem of the low efficiency and possible local optimum (e.g., large static imbalance) of the iteration of two steps in Section 3.3, we propose Property 3 and dynamic adjustment strategy for QPDAA.

4.1. Related Property. For optimizing the static imbalance of the layout scheme, we introduce Property 3.

Property 3. Assume $A_{1}\left(x_{m}, y_{m}\right)$ is the mass center of an orthogonal packing scheme $\mathbf{X}_{1}$ of this problem and $A_{1}\left(x_{m}, y_{m}\right) \neq(0,0)$ and $\mathbf{X}_{2}$ is another scheme obtained by interchanging centers of two rectangles $R_{i}$ with a mass $m_{i}$ and $R_{j}\left(i, j \in \mathbf{I}_{n}\right.$ and $\left.i \neq j\right)$ with a mass $m_{j}$ in $\mathbf{X}_{1}$. If $m_{j}>m_{i}$, $d_{j}>d_{i}$, centers $\left(x_{i}, y_{i}\right)$ and $\left(x_{i}, y_{i}\right)$ satisfy Formula (17), then $J\left(\mathbf{X}_{1}\right)>J\left(\mathbf{X}_{2}\right)$, where $d_{k}=\left(x_{k}^{2}+y_{k}^{2}\right)^{1 / 2}(k=i, j)$ :

$2\left(x_{j}-x_{i}\right) x_{m} \sum_{k=1}^{n} m_{k}+2\left(y_{j}-y_{i}\right) y_{m} \sum_{k=1}^{n} m_{k}>\left(m_{j}-m_{i}\right) d_{i j}^{2}$.

In Formula (17), $d_{i j}=\left(\left(x_{j}-x_{i}\right)^{2}+\left(y_{j}-y_{i}\right)^{2}\right)^{1 / 2}$.

Proof. Consider

$$
\begin{aligned}
\because J^{2}\left(X_{1}\right) & =\left(\sum_{k=1}^{n} m_{k} x_{k}\right)^{2}+\left(\sum_{k=1}^{n} m_{k} y_{k}\right)^{2} \\
J^{2}\left(X_{2}\right)= & \left(\sum_{k=1}^{n} m_{k} x_{k}-m_{i} x_{i}-m_{j} x_{j}+m_{i} x_{j}+m_{j} x_{i}\right)^{2} \\
& +\left(\sum_{k=1}^{n} m_{k} y_{k}-m_{i} y_{i}-m_{j} y_{j}+m_{i} y_{j}+m_{j} y_{i}\right)^{2}
\end{aligned}
$$

$$
\begin{aligned}
\therefore J^{2} & \left(X_{1}\right)-J^{2}\left(X_{2}\right) \\
= & \left(m_{i} x_{i}+m_{j} x_{j}-m_{i} x_{j}-m_{j} x_{i}\right) \\
& \times\left(2 \sum_{k=1}^{n} m_{k} x_{k}-m_{i} x_{i}-m_{j} x_{j}+m_{i} x_{j}+m_{j} x_{i}\right) \\
& +\left(m_{i} y_{i}+m_{j} y_{j}-m_{i} y_{j}-m_{j} y_{i}\right) \\
& \times\left(2 \sum_{k=1}^{n} m_{k} y_{k}-m_{i} y_{i}-m_{j} y_{j}+m_{i} y_{j}+m_{j} y_{i}\right)^{2} \\
= & \left(m_{i}-m_{j}\right)\left(x_{i}-x_{j}\right) \\
& \times\left[2 x_{m} \sum_{k=1}^{n} m_{k} x_{k}-\left(m_{i}-m_{j}\right)\left(x_{i}-x_{j}\right)\right] \\
& +\left(m_{i}-m_{j}\right)\left(y_{i}-y_{j}\right) \\
& \times\left[2 y_{m} \sum_{k=1}^{n} m_{k} y_{k}-\left(m_{i}-m_{j}\right)\left(y_{i}-y_{j}\right)\right] \\
= & 2\left(x_{j}-x_{i}\right) x_{m} \sum_{k=1}^{n} m_{k}+2\left(y_{j}-y_{i}\right) y_{m} \sum_{k=1}^{n} m_{k} \\
& \left(m_{i}\right) d_{i j}^{2}>0 .
\end{aligned}
$$

Property 3 indicates that, for selecting rectangle $R_{i} \in \mathbf{X}_{1}$, we can find a rectangle $R_{j}$ in the sector area $A_{1} O A_{2}$ with an angle $\varphi$ (shown as in Figure 11) and interchange them to obtain $\mathbf{X}_{2}$ whose static imbalance is less than $\mathbf{X}_{1}$. Here, the angle $\varphi$ satisfies

$$
\begin{aligned}
& 2 \sum_{k=1}^{n} m_{k}\left(x_{m}^{2}+y_{m}^{2}\right)^{1 / 2}\left[\left(x_{j}-x_{i}\right)^{2}+\left(y_{j}-y_{i}\right)^{2}\right]^{1 / 2} \cos (\varphi) \\
& \quad=\left(m_{j}-m_{i}\right) d_{i j}^{2}
\end{aligned}
$$

4.2. Dynamic Adjustment Strategy. Let $\mathbf{X}=\left(x_{k}, y_{k}, \theta_{k} \mid k=\right.$ $1,2, \ldots, n)$; we consider the following dynamic adjustment strategy. 
TABLE 2: Performance comparisons of four algorithms.

\begin{tabular}{|c|c|c|c|c|c|c|c|}
\hline Example number & Size & Algorithm & Average $r$ & Standard deviation & Minimal $r$ & Maximal $r$ & Average $t / s$ \\
\hline \multirow{3}{*}{1} & \multirow{3}{*}{5} & GA + HA [35] & 11.7703 & 0.0335 & 11.7597 & 11.8655 & 0.242 \\
\hline & & CA-PSLS [34] & 12.2181 & 0.5214 & 11.5443 & 13.0982 & 11.657 \\
\hline & & QPDAA & 11.6756 & 0.0235 & 11.4737 & 11.8533 & 0.953 \\
\hline \multirow{3}{*}{2} & \multirow{3}{*}{6} & IGA [44] & - & - & 14.62000 & - & 18.000 \\
\hline & & CA-PSLS [34] & 15.19402 & - & 14.39625 & - & 19.904 \\
\hline & & QPDAA & 14.68374 & - & 14.344996 & 14.909841 & 4.963 \\
\hline \multirow{3}{*}{3} & \multirow{3}{*}{9} & $\mathrm{GA}+\mathrm{HA}$ [35] & 18.1709 & 0.2361 & 17.8988 & 18.6041 & 1.262 \\
\hline & & CA-PSLS [34] & 20.0899 & 1.1957 & 18.3758 & 22.0803 & 58.126 \\
\hline & & QPDAA & 18.2461 & 0.0694 & 17.6880 & 18.5983 & 1.110 \\
\hline \multirow{3}{*}{4} & \multirow{3}{*}{20} & $\mathrm{GA}+\mathrm{HA}[35]$ & 23.2713 & 0.4728 & 22.6396 & 24.0605 & 9.989 \\
\hline & & CA-PSLS [34] & 32.7216 & 4.0065 & 27.2863 & 38.5048 & 321.071 \\
\hline & & QPDAA & 22.8368 & 0.0222 & 22.3510 & 23.7884 & 2.625 \\
\hline \multirow{3}{*}{5} & \multirow{3}{*}{40} & GA [35] & 119.2396 & 1.5087 & 115.836 & 120.7099 & 62.795 \\
\hline & & CA-PSLS [34] & 253.4165 & 43.8096 & 195.5914 & 306.2740 & 1367.573 \\
\hline & & QPDAA & 119.1383 & 0.6887 & 115.6252 & 120.5102 & 3.375 \\
\hline
\end{tabular}

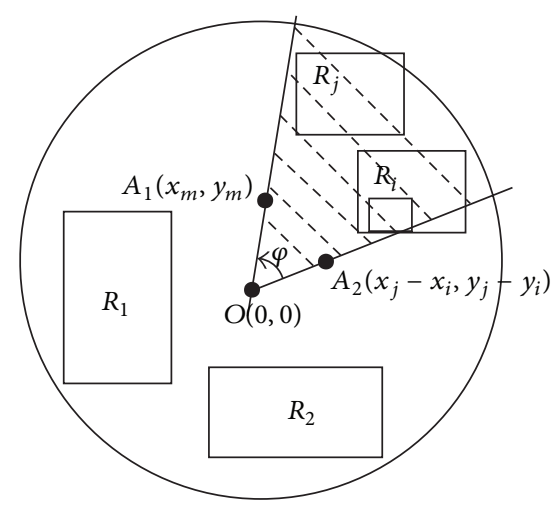

FIGURE 11: The geometric area of Formula (17).

TABLE 3: The layout schemes of the proposed QPDAA for Examples 1 and 2.

\begin{tabular}{cccccc}
\hline & \multicolumn{2}{c}{ Example 1} & \multicolumn{3}{c}{ Example 2} \\
$i$ & $x_{i}$ & $y_{i}$ & $i$ & $x_{i}$ & $y_{i}$ \\
\hline 1 & 5.017966 & -4.093750 & 1 & -5.810001 & -7.386930 \\
2 & 4.932835 & 2.906250 & 2 & 5.686532 & 6.537550 \\
3 & -3.982034 & -4.093750 & 3 & 8.827469 & -0.519116 \\
4 & -5.067165 & 0.906250 & 4 & 3.371405 & -7.538003 \\
5 & -2.067165 & 5.906250 & 5 & -3.346021 & 6.667169 \\
& & & 6 & -7.636339 & -1.338337 \\
\hline
\end{tabular}

4.2.1. Rectangle-Interchanging. According to Property 3, the static imbalance of the packing scheme can be decreased by interchanging positions of two rectangles.

Two rectangles, $R_{i}$ with a smaller mass and $R_{j}$ with a larger mass $\left(i, j \in \mathbf{I}_{n}, d_{i}<0.5 R_{0}, 0.5 R_{0}<d_{j}<R_{0}\right)$, are selected from $\mathbf{X}$. If $R_{i}$ and $R_{j}$ satisfy Formula (17), then we update $\mathbf{X}$ by $x_{i} \leftrightarrow x_{j}, y_{i} \leftrightarrow y_{j}, \theta_{i} \leftrightarrow \theta_{j}$.
TABLE 4: The layout schemes of the proposed QPDAA for Example 3.

\begin{tabular}{ccc}
\hline$i$ & $x_{i}$ & $y_{i}$ \\
\hline 1 & 0.074698 & -3.832386 \\
2 & 2.329571 & 3.285391 \\
3 & 4.944505 & -10.373944 \\
4 & 3.514680 & 11.503878 \\
5 & 11.343280 & 1.762618 \\
6 & -6.074076 & -9.903838 \\
7 & 10.077353 & -5.359918 \\
8 & -10.295647 & -2.839607 \\
9 & -7.690402 & 6.187573 \\
\hline
\end{tabular}

4.2.2. Rotation and Off-Trap. (i) A rectangle with a larger pain degree is found out from $\mathbf{X}$ and is rotated $90^{\circ}$ round its center counterclockwise direction to relieve its pain. (ii) A rectangle with a larger pain degree is found out from $\mathbf{X}$ and is moved to such a place in the container where its pain degree is smaller. 
TABLE 5: The layout scheme of the proposed QPDAA for Example 4.

\begin{tabular}{lcc}
\hline$i$ & $x_{i}$ & $y_{i}$ \\
\hline 1 & 2.275791 & -8.061370 \\
2 & 0.693375 & 15.930070 \\
3 & 8.143939 & 8.438630 \\
4 & -4.806625 & 15.930070 \\
5 & -5.021757 & -17.061370 \\
6 & 5.978243 & -15.573636 \\
7 & 0.275791 & -3.561370 \\
8 & -12.856061 & 7.930070 \\
9 & -5.724209 & -10.561370 \\
10 & 6.193375 & 17.938630 \\
11 & -16.608718 & 1.930070 \\
12 & 7.143939 & 3.938630 \\
13 & -7.608718 & 1.930070 \\
14 & 16.143939 & 1.426364 \\
15 & -1.856061 & 8.430070 \\
16 & 9.143939 & 13.938630 \\
17 & 3.391282 & 0.438630 \\
18 & -14.724209 & -5.069930 \\
19 & 12.275791 & -7.573636 \\
20 & -12.724209 & -12.069930 \\
\hline & &
\end{tabular}

We know that the role of (ii) is similar to a construction of the nonisomorphic layout pattern $[42,43]$.

4.2.3. Center Translation. If its mass center $P_{m}\left(x_{m}, y_{m}\right) \neq$ $O(0,0)$, then $\mathbf{X}=\left(x_{k}-x_{m}, y_{k}-y_{m}, \theta_{k} \mid k=1,2, \ldots, n\right)$.

\section{The Proposed Algorithm}

Through an organic combination of the compact and feasible solution strategy and dynamic adjustment strategy, we present QPDAA for BCOURP.

Let $R_{0}$ and $r^{*}$ be the predetermined value and an allowable maximum of the envelope radius of the solution, respectively; $n$ is the number of rectangles. $P_{m}\left(x_{m}, y_{m}\right), f(\mathbf{X})$, and $E(\mathbf{X})$ denote the mass center, envelope radius, and extruded potential energy of the packing scheme $\mathbf{X}$, respectively. $E_{k}(\mathbf{X})(k=1,2, \ldots, n)$ is the extruded potential energy of the rectangle $R_{k} ; h$ is the step length; $N$ is the maximum translation times. Then steps of the proposed QPDAA are shown in Algorithm 1.

\section{Experiments and Analysis}

6.1. Experiments. The proposed QPDAA is coded in $\mathrm{VC}++$ 6.0 and carried on a Pentium $3 \mathrm{GHZ}$ PC with $512 \mathrm{MB}$ memory. CA-PSLS [34], GA-HA [35] are coded in $\mathrm{VC}++6.0$ and two algorithms are carried on a Pentium 1.83 GHZ with $512 \mathrm{MB}$ memory; IGA [44] is carried on an IBM $586166 \mathrm{MHz}$.

Experiment 1. Five examples are taken from [33, 35] and are used in testing the performance of the proposed QPDAA.
TABLE 6: The layout scheme of the proposed QPDAA for Example 5.

\begin{tabular}{|c|c|c|}
\hline$i$ & $x_{i}$ & $y_{i}$ \\
\hline 1 & -20.484058 & -97.670716 \\
\hline 2 & 96.802177 & -23.135827 \\
\hline 3 & -32.957579 & -3.670716 \\
\hline 4 & -17.484058 & -67.670716 \\
\hline 5 & -34.765848 & 92.329284 \\
\hline 6 & -16.197823 & -34.670716 \\
\hline 7 & 49.515942 & -85.946702 \\
\hline 8 & -5.847798 & 30.463901 \\
\hline 9 & 24.515942 & -86.536099 \\
\hline 10 & -55.957579 & -24.941525 \\
\hline 11 & -93.957579 & 1.531453 \\
\hline 12 & 22.042421 & 4.053298 \\
\hline 13 & 23.234152 & 85.053298 \\
\hline 14 & 71.802177 & -25.946702 \\
\hline 15 & -71.484058 & -59.468547 \\
\hline 16 & 92.042421 & 9.864173 \\
\hline 17 & 23.152202 & 57.053298 \\
\hline 18 & 3.515942 & -83.536099 \\
\hline 19 & -32.847798 & 65.329284 \\
\hline 20 & -41.484058 & -57.941525 \\
\hline 21 & -32.957579 & 30.329284 \\
\hline 22 & 66.042421 & 1.053298 \\
\hline 23 & 9.802177 & -56.536099 \\
\hline 24 & -4.765848 & 88.463901 \\
\hline 25 & -59.957579 & 4.058475 \\
\hline 26 & -4.847798 & 59.463901 \\
\hline 27 & 12.802177 & -29.536099 \\
\hline 28 & -86.957579 & -30.468547 \\
\hline 29 & 52.515942 & -63.946702 \\
\hline 30 & -64.765848 & 69.531453 \\
\hline 31 & 44.802177 & -32.946702 \\
\hline 32 & -85.957579 & 35.531453 \\
\hline 33 & 86.152202 & 40.864173 \\
\hline 34 & 57.234152 & 69.864173 \\
\hline 35 & 21.152202 & 32.053298 \\
\hline 36 & -6.957579 & -0.536099 \\
\hline 37 & -56.957579 & 25.058475 \\
\hline 38 & -45.484058 & -85.941525 \\
\hline 39 & 53.152202 & 35.053298 \\
\hline 40 & 45.042421 & 5.053298 \\
\hline
\end{tabular}

Data of all examples are shown in Table 1. For the proposed algorithm, we take $\alpha=18, \beta=56, \varepsilon=10^{-20}, h=0.8$, $\delta=6$, and $N=120$, respectively. For Examples $1-5$, we take $R_{0}=11.4,14.3,17.5,22.3$, and 115.5 and take $r^{*}=1.05 R_{0}$, $1.05 R_{0}, 1.07 R_{0}, 1.07 R_{0}$, and $1.05 R_{0}$, respectively. Running the proposed QPDAA 30 times for each example (the success rate is $100 \%$ ), we show its average running time, average envelope radius, standard variance of the radius, and the maximum and minimum envelope radii in Table 2; for each example, its layout scheme diagraph is shown in Figure 12; the other data 
TABLE 7: The effect of parameters $R_{0}$ and $r^{*}$ on the optimal radius and running time for the proposed QPDAA.

\begin{tabular}{|c|c|c|c|c|c|}
\hline Experiment number & 1 & 2 & 3 & 4 & 5 \\
\hline Size & 5 & 6 & 9 & 20 & 40 \\
\hline$R_{0}$ & 11.4 & 14.3 & 17.5 & 22.3 & 115.5 \\
\hline$r^{*}$ & $1.05 R_{0}$ & $1.05 R_{0}$ & $1.07 R_{0}$ & $1.07 R_{0}$ & $1.05 R_{0}$ \\
\hline The optimal radius & 11.4737 & 14.344996 & 17.6880 & 22.3510 & 115.6252 \\
\hline Running time & 0.953 & 3.5872 & 1.110 & 2.625 & 2.625 \\
\hline$R_{0}$ & 11.4 & 14.3 & 17.5 & 22.06 & 114.8 \\
\hline$r^{*}$ & $1.02 R_{0}$ & $1.02 R_{0}$ & $1.03 R_{0}$ & $1.01 R_{0}$ & $1.01 R_{0}$ \\
\hline The optimal radius & 11.450458 & 14.309924 & 17.597856 & 22.141009 & 115.042385 \\
\hline Running time & 1.297 & 8.963 & 7.906 & 12.920 & 17.125 \\
\hline
\end{tabular}

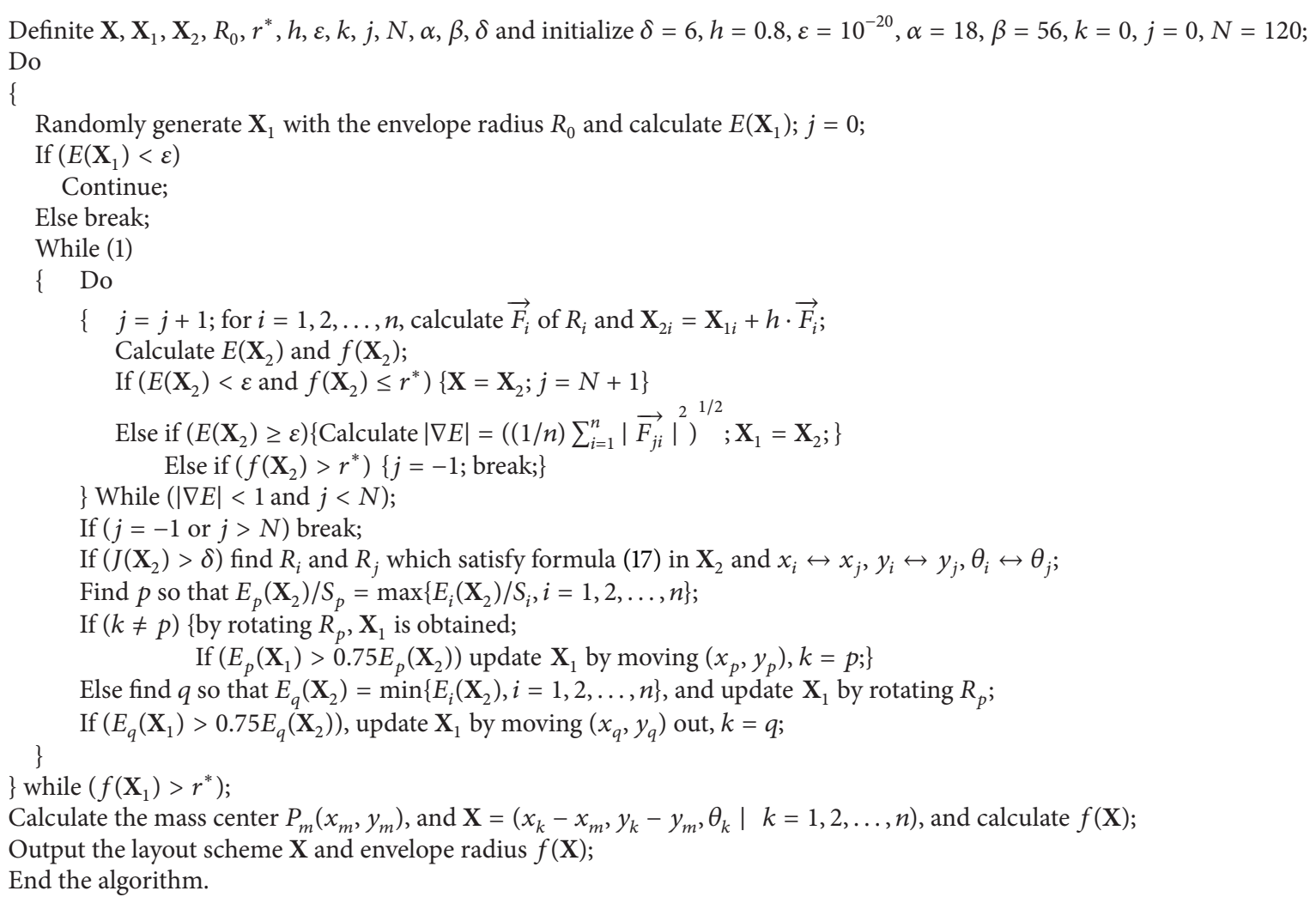

Algorithm 1

TABLE 8: The optimal layout schemes of Examples 1 and 2 for the proposed QPDAA.

\begin{tabular}{cccccc}
\hline$i$ & $x_{i}$ & $y_{i}$ & $i$ & $x_{i}$ & $y_{i}$ \\
\hline 1 & -2.954693 & -5.470039 & 1 & 4.948526 & 8.054106 \\
2 & 5.045307 & -2.429991 & 2 & 4.903437 & -6.945894 \\
3 & -2.408844 & 4.570009 & 3 & -4.140424 & -8.010294 \\
4 & -4.954693 & -0.429991 & 4 & 5.911271 & 1.054106 \\
5 & 5.591156 & 4.570009 & 5 & -4.088729 & 5.989706 \\
& & & 6 & -5.096563 & -2.010294 \\
\hline
\end{tabular}

in Table 2 is taken from [33-35]. The optimal layout schemes of the proposed QPDAA are shown in Tables 3, 4, 5, and 6 for 5 examples.

Experiment 2. For testing the effects of $R_{0}$ and $r^{*}$ on the minimal radius and running time with the proposed QPDAA, we take another set of $R_{0}$ and $r^{*}$ (five examples and other parameters are the same as those of Experiment 1) and run the proposed QPDAA procedure 30 times for each example. The minimal radii and running times are given in Table 7. Their layout schemes and layout diagraphs are shown in Tables 8, 9, 10, and 11 and Figure 13. It can be found from 


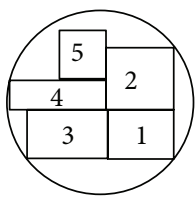

(a)

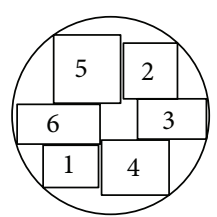

(b)

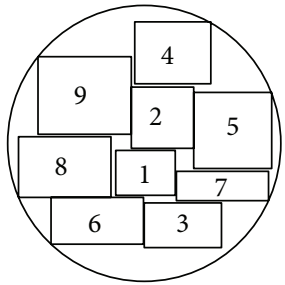

(c)

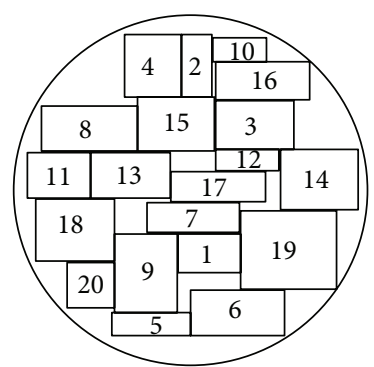

(d)

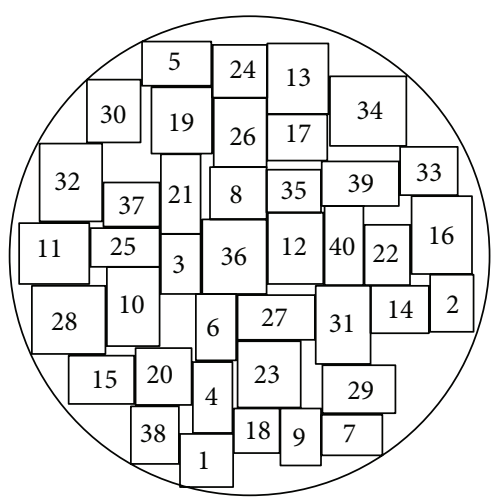

(e)

FIGURE 12: The layout diagraphs of five examples for the proposed QPDAA.

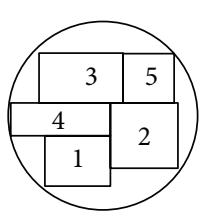

(a)

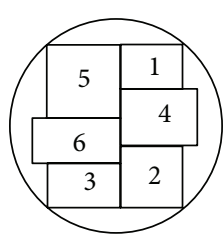

(b)

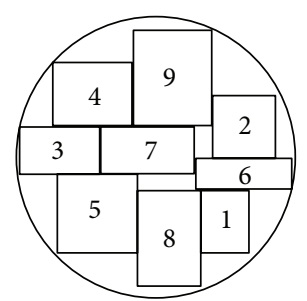

(c)

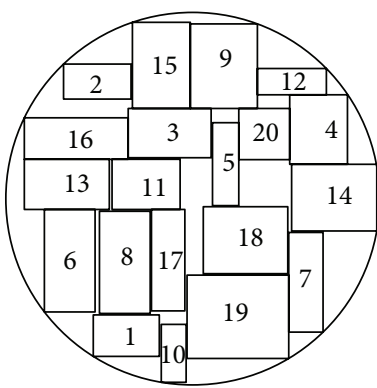

(d)

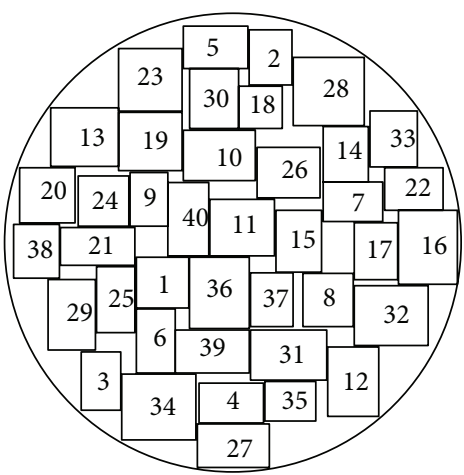

(e)

FIGURE 13: The optimal layout scheme diagraphs of five examples for the proposed QPDAA.

TABLE 9: The optimal layout schemes of Example 3 for the proposed QPDAA.

\begin{tabular}{ccc}
\hline$i$ & $x_{i}$ & $y_{i}$ \\
\hline 1 & 8.952825 & -8.127957 \\
2 & 11.192355 & 3.872043 \\
3 & -11.893145 & 0.866506 \\
4 & -7.807645 & 7.896834 \\
5 & -7.161996 & -7.133494 \\
6 & 11.084588 & -2.235259 \\
7 & -0.893145 & 0.866506 \\
8 & 1.838004 & -10.127957 \\
9 & 2.125081 & 10.168689 \\
\hline
\end{tabular}

Table 7 that changing values of $R_{0}$ and $r^{*}$ we can obtain the packing scheme with a smaller envelop radius, but it costs more time for each example. So, values $R_{0}$ and $r^{*}$ in Experiment 1 can be applied to make a tradeoff between the computational effectiveness and solution quality.

In order to further test the effectiveness of the proposed QPDAA, we consider Experiment 3.
Experiment 3. Numbers of rectangles (generated randomly) of three examples are 50,55, and 60 and their lengths and widths are between 20 and 40. For the proposed QPDAA, we take $\alpha=18, \beta=56, \varepsilon=10^{-20}, h=0.8, \delta=10$, $N=120, R_{0}=125.9,133.8,137.2$, and $r^{*}=126.8,134.6,138.8$ for Examples 1-3, respectively. For GA + HA, the population size, mutation probability, and max number of the iteration are $30,0.125$, and 50, respectively. By running $\mathrm{HA}+\mathrm{GA}$ and the proposed QPDAA 30 times for three examples, respectively, their optimal envelop radii and average times are shown in Table 12, respectively. The optimal packing scheme diagrams of GA + HA and the proposed QPDAA are shown in Figures 14(a)-14(c) and Figures 14(d)-14(f). We can know from Table 12 that both the solution quality and computational quality of the proposed QPDAA are obviously higher than those of GA + HA.

Note that, in Experiment 3, the procedure of GA + HA is coded by author and is carried on a Pentium $3 \mathrm{GHZ}$ with $512 \mathrm{MB}$ memory.

6.2. Analysis. From data of Tables 2, 7, and 12, we know that the solution quality of the proposed QPDAA algorithm is higher than those of CA-PSLS and GA-HA. Compared 


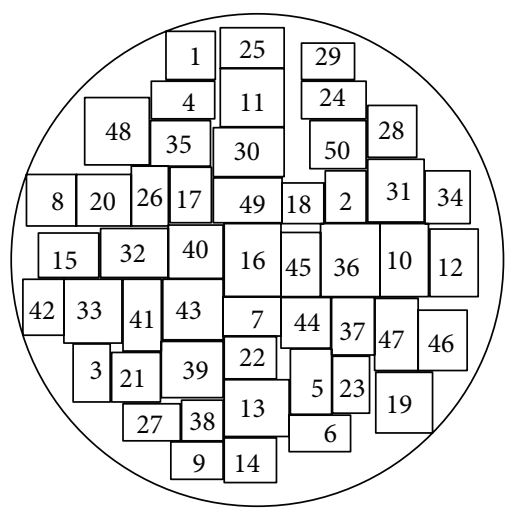

(a) GA_HA for 50 rectangles

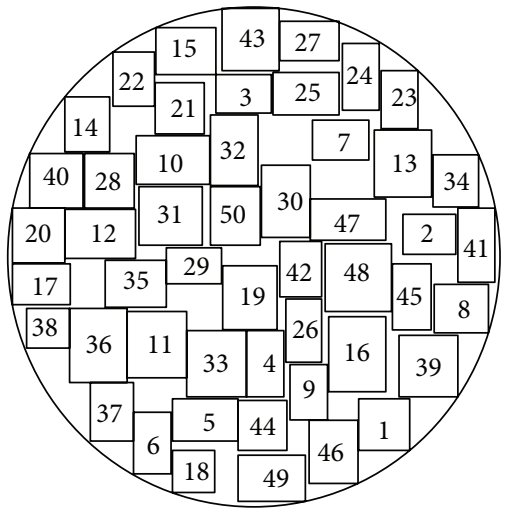

(d) QPDAA for 50 rectangles

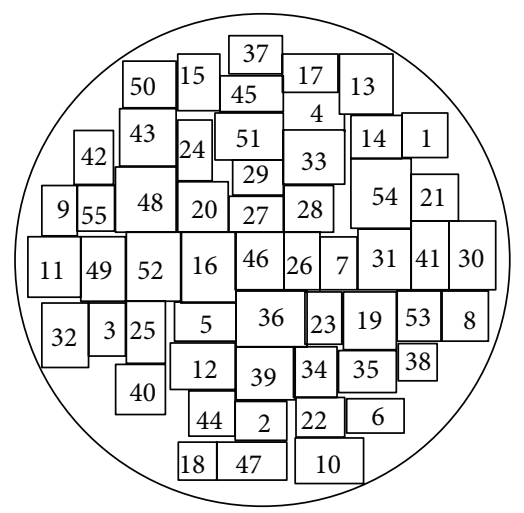

(b) GA_HA for 55 rectangles

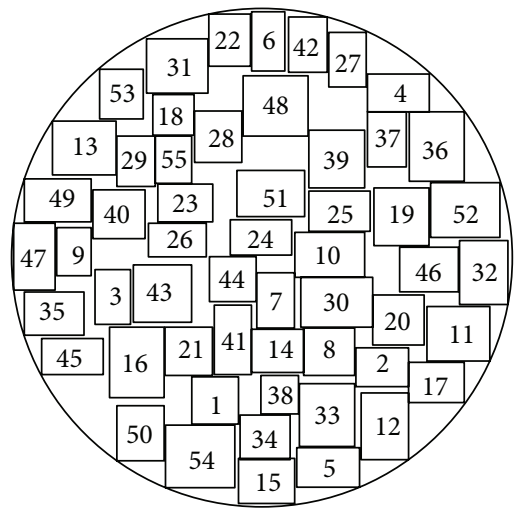

(e) QPDAA for 55 rectangles

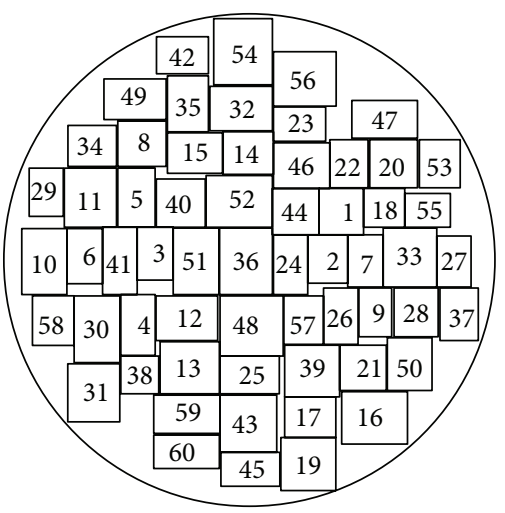

(c) GA_HA for 60 rectangles

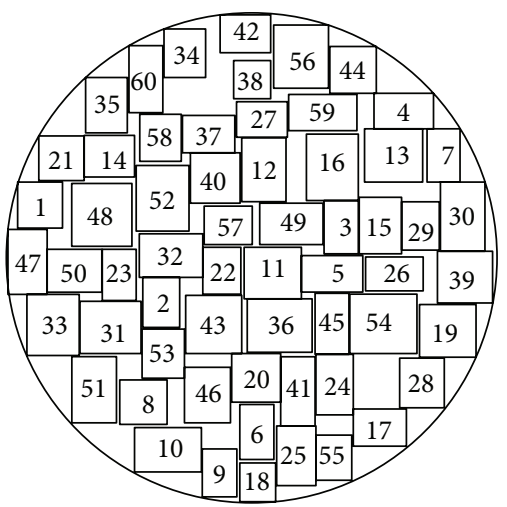

(f) QPDAA for 60 rectangles

FIGURE 14: Packing scheme diagrams of GA + HA and the proposed QPDAA for Experiment 3.

with those of CA-PSLS, embedded degree functions of the proposed QPDAA can make the layout scheme more compact. Due to the fixed candidate positions of GA + HA, it is difficult to find the best position for some rectangles close to the marginal region of the container. The deficiency of a mechanism of decreasing the static balance in the process of packing rectangles also limits the solution quality of GA + HA. The experimental results illustrate the effectiveness of the proposed QPDAA.

The computational efficiency of the proposed QPDAA is one magnitude higher than those of CA-PSLS. There are two reasons. (i) Owing to orthogonal packing, searching the optimal solution in the 2D solution of the proposed QPDAA is easier than that in the 3D solution space of CA-PSLS. (ii) In order to improve the solution quality of CA-PSLS, PSO is used to optimize the feasible solution obtained through the gradient method based on two discontinuous embedded degree functions, but the proposed QPDAA need not do the PSO optimization without reducing its solution quality. Except for Example 1, the computational efficiency of the proposed QPDAA is higher than that of GA + HA. And with the increase of the size of the packing problem, the advantage of the proposed QPDAA is more obvious. This is because computational complexities of the extruded resultant force and potential energy are $O(n)$ in this paper, but the computational complexity of noninterference judgment is $O\left(n^{2}\right)$. In addition, for GA + HA, with the increasing of the number of rectangles, the number of candidate positions of each rectangle increases dramatically. These reasons lead to the computational efficiency of the proposed QPDAA higher than that of GA + HA for the BCOURP with a large size.

\section{Conclusions}

Taking the layout design of a satellite module as the application background, we have proposed the QPDAA for the BCOURP problem in this paper. Two continuous embedded functions between orthogonal rectangles and between the rectangle and container are constructed to overcome the weakness of embedded functions in [34]. And the suggestion of the extruded resultant force formula and the potential energy function of the rectangle packing system based on the proposed embedded functions make solving the BCOURP problem simple and effective as solving the circle packing problem [37-40]. The proposed dynamic adjustment strategy can quickly decrease the static imbalance of the packing scheme and make the iteration skip the local optimum. The experiment results show that the proposed QPDAA is superior to existing algorithms in performance for the BCOURP problem, especially for the BCOURP problem with the large size. The next work is to extend the above algorithm into solving the $3 \mathrm{D}$ satellite module payload packing problem. 
TABLE 10: The optimal layout scheme of Example 4 for the proposed QPDAA.

\begin{tabular}{lcc}
\hline$i$ & $x_{i}$ & $y_{i}$ \\
\hline 1 & -7.913018 & -16.1629110 \\
2 & -11.4301555 & 13.766710 \\
3 & -2.797531 & 7.599405 \\
4 & 14.854397 & 8.176985 \\
5 & 3.751568 & 4.136671 \\
6 & -14.476432 & -7.582581 \\
7 & 13.305438 & -9.941647 \\
8 & -8.088653 & -7.608034 \\
9 & 3.747621 & 15.611128 \\
10 & -2.290583 & -18.311460 \\
11 & -5.543514 & 1.554382 \\
12 & 11.758080 & 13.883226 \\
13 & -14.800586 & 1.537640 \\
14 & 16.662225 & 0.074950 \\
15 & -3.889690 & 15.762332 \\
16 & -13.867726 & 7.136671 \\
17 & -2.96 .2478 & -7.489430 \\
18 & 6.210196 & -4.937357 \\
19 & 5.257463 & -14.032250 \\
20 & 8.273430 & 7.563706 \\
\hline & &
\end{tabular}

\section{Appendices}

\section{A. HA + GA [35]}

Input the length, width, and mass of the rectangle $F_{i}(i=$ $1,2, \ldots, n)$ in turn, initialize the number $N$ of the maximal iteration times, and generate their placing sequence set $\{t(i), i=1,2, \ldots, n\}$.

Step 1. Set $k=1$.

Step 2. Set the center of $F_{t(1)}$ at $(0,0)$ and its long side is parallel to $x_{1}$-axis, $i=2, j=1$.

Step 3. For $j=1,2, \ldots, i-1$, calculate centers and direction angles of 16 candidate positions (see Figure 1) of the rectangle $F_{t(i)}$ with respect to the rectangle $F_{t(j)}$. From its candidate positions eliminate unfeasible ones and calculate the optimal one (i.e., compared with other feasible candidate positions, it makes the packing scheme of the first $i$ rectangles have less envelop radius).

Step 4. If $i<n$ then update the current packing scheme, $i++$, and go to Step 3; otherwise, go to Step 5.

Step 5. If $k<N$ then update the optimal packing scheme and use GA to generate a new placing sequence set $\{t(i), i=$ $1,2, \ldots, n\}$ and go to Step 2; otherwise, go to Step 6 .

Step 6. Output the optimal packing scheme and envelop radius; algorithm ends.
TABLE 11: The optimal layout scheme of Example 5 for the proposed QPDAA.

\begin{tabular}{|c|c|c|}
\hline$i$ & $x_{i}$ & $y_{i}$ \\
\hline 1 & -35.716569 & -20.219336 \\
\hline 2 & 19.295868 & 92.929139 \\
\hline 3 & -66.464390 & -69.791544 \\
\hline 4 & -0.799905 & -80.612101 \\
\hline 5 & -8.704132 & 97.929139 \\
\hline 6 & -38.754325 & -49.219336 \\
\hline 7 & 60.543358 & 20.832873 \\
\hline 8 & 47.933196 & -29.082316 \\
\hline 9 & -42.456642 & 21.911290 \\
\hline 10 & -6.576451 & 43.929139 \\
\hline 11 & 4.543358 & 7.350623 \\
\hline 12 & 60.568942 & -69.995791 \\
\hline 13 & -74.792262 & 52.886007 \\
\hline 14 & 57.004507 & 44.832873 \\
\hline 15 & 33.5433588 & 0.917684 \\
\hline 16 & 97.933196 & -2.928148 \\
\hline 17 & 71.933196 & -4.167127 \\
\hline 18 & 14.886008 & 67.929139 \\
\hline 19 & -41.5764518 & 50.911290 \\
\hline 20 & -93.27781098 & 23.736930 \\
\hline 21 & -67.7165699 & -2.088710 \\
\hline 22 & 90.543358 & 27.071852 \\
\hline 23 & -41.704132 & 81.911290 \\
\hline 24 & -65.456642 & 20.911290 \\
\hline 25 & -58.7543259 & -29.088710 \\
\hline 26 & 28.423549 & 35.350623 \\
\hline 27 & 0.535610 & -101.612101 \\
\hline 28 & 48.2958689 & 75.832873 \\
\hline 29 & -80.754325 & -36.263070 \\
\hline 30 & -9.113992 & 71.929139 \\
\hline 31 & 28.245675 & -56.649377 \\
\hline 32 & 79.933196 & -36.928148 \\
\hline 33 & 81.004507 & 52.071852 \\
\hline 34 & -37.464390 & -82.649377 \\
\hline 35 & 29.200095 & -79.649377 \\
\hline 36 & -6.716569 & -25.649377 \\
\hline 37 & 20.283431 & -29.082316 \\
\hline 38 & -98.716569 & -4.263070 \\
\hline 39 & -9.754325 & -54.649377 \\
\hline 40 & -22.456642 & 11.780664 \\
\hline
\end{tabular}

\section{B. Results of Experiment 2}

See Tables 8, 9, 10, and 11 and Figure 13.

\section{Conflict of Interests}

The authors declare that there is no conflict of interests regarding the publication of this paper. 
TABLE 12: The effect of $R_{0}$ and $r^{*}$ on the optimal radius and running time for the proposed QPDAA.

\begin{tabular}{lccccc}
\hline \multirow{2}{*}{ Example number } & \multirow{2}{*}{ Size } & \multicolumn{2}{c}{ HA + GA } & \multicolumn{2}{c}{ QPDAA } \\
& & The optimal radius & Running time (s) & The optimal radius & Running time (s) \\
\hline 1 & 50 & 127.667244 & 30.170088 & 126.567311 & 13.345431 \\
2 & 55 & 134.844782 & 42.654031 & 134.105091 & 19.056438 \\
3 & 60 & 139.072606 & 66.908203 & 137.833657 & 37.01790 \\
\hline
\end{tabular}

\section{Acknowledgments}

This work is supported by the National Natural Science Foundation of China (Grant no. 61272294) and Research Foundation of Education Bureau of Hunan Province, China (Grant no. 11A120), and the Construct Program of the Key Discipline in Hunan Province. And the authors are also grateful to the anonymous referees for their advice and reviews for this paper.

\section{References}

[1] K. He, W. Huang, and Y. Jin, "An efficient deterministic heuristic for two-dimensional rectangular packing," Computers \& Operations Research, vol. 39, no. 7, pp. 1355-1363, 2012.

[2] N. Lesh, J. Marks, A. McMahon, and M. Mitzenmacher, "Exhaustive approaches to 2D rectangular perfect packings," Information Processing Letters, vol. 90, no. 1, pp. 7-14, 2004.

[3] E.-M. Feng and X.-L. Wang, "An optimization model for the layout of a group of cuboids in a satellite module and its global optimization algorithm," Operation Research Transaction, vol. 5, no. 3, pp. 71-77, 2001 (Chinese).

[4] J. Roth and R. Hasimshony, "Comparison of existing threeroom apartment plans with computer-generated layouts," Planning and Design, vol. 14, no. 2, pp. 149-161, 1987.

[5] J. Leung, "A new graph-theoretic heuristic for facility layout," Management Science, vol. 38, no. 4, pp. 594-606, 1992.

[6] J. M. V. de Carvalho, "Exact solution of bin-packing problems using column generation and branch-and-bound," Annals of Operations Research, vol. 86, pp. 629-659, 1999.

[7] R. Macedo, C. Alves, and J. M. V. de Carvalho, "Arc-flow model for the two-dimensional guillotine cutting stock problem," Computers and Operations Research, vol. 37, no. 6, pp. 991-1001, 2010.

[8] Y.-D. Cui, C. L. Zhang, and Y. Zhao, "A continued fractions and branch-and-bound algorithm for generating cutting patterns with equal rectangles," Journal of Computer-Aided Design \& Computer Graphics, vol. 16, no. 2, pp. 252-256, 2004.

[9] F. Clautiaux, A. Jouglet, J. Carlier, and A. Moukrim, "A new constraint programming approach for the orthogonal packing problem," Computers \& Operations Research, vol. 35, no. 3, pp. 944-959, 2008.

[10] K. Yoon, S. Ahn, and M. Kang, "An improved best-first branchand-bound algorithm for constrained two-dimensional guillotine cutting problems," International Journal of Production Research, vol. 51, no. 6, pp. 1680-1693, 2013.

[11] E. G. Birgin, R. D. Lobato, and R. Morabito, "Generating unconstrained two-dimensional non-guillotine cutting patterns by a recursive partitioning algorithm," Journal of the Operational Research Society, vol. 63, no. 2, pp. 183-200, 2012.

[12] Y.-L. Wu, W. Huang, S.-C. Lau, C. Wong, and G. H. Young, "An effective quasi-human based heuristic for solving the rectangle packing problem," European Journal of Operational Research, vol. 141, no. 2, pp. 341-358, 2002.

[13] D.-F. Zhang, S.-H. Han, and W.-G. Ye, "Bricklaying heuristic algorithm for the orthogonal rectangular packing problem," Chinese Journal of Computers, vol. 31, no. 3, pp. 509-514, 2008.

[14] R. E. Korf, M. D. Moffitt, and M. E. Pollack, "Optimal rectangle packing," Annals of Operations Research, vol. 179, no. 1, pp. 261295, 2010.

[15] C. Charalambous and K. Fleszar, "A constructive bin-oriented heuristic for the two-dimensional bin packing problem with guillotine cuts," Computers and Operations Research, vol. 38, no. 10, pp. 1443-1451, 2011.

[16] S. Polyakovsky and R. M'Hallah, "An agent-based approach to the two-dimensional guillotine bin packing problem," European Journal of Operational Research, vol. 192, no. 3, pp. 767-781, 2009.

[17] S. Khebbache1, C. Prins, and A. Yalaoui, "Iterated local search algorithm for the constrained two-dimensional non-guillotine cutting problem," Journal of Industrial and Systems Engineering, vol. 2, no. 3, pp. 164-179, 2008.

[18] J. F. Gonçalves and M. G. C. Resende, "A parallel multi-population genetic algorithm for a constrained two-dimensional orthogonal packing problem," Journal of Combinatorial Optimization, vol. 22, no. 2, pp. 180-201, 2011.

[19] E. G. Birgin, R. D. Lobato, and R. Morabito, "An effective recursive partitioning approach for the packing of identical rectangles in a rectangle," Journal of the Operational Research Society, vol. 61, no. 2, pp. 306-320, 2010.

[20] M. Dolatabadi, A. Lodi, and M. Monaci, "Exact algorithms for the two-dimensional guillotine knapsack," Computers and Operations Research, vol. 39, no. 1, pp. 48-53, 2012.

[21] S. Martello, M. Monaci, and D. Vigo, "An exact approach to the strip-packing problem," INFORMS Journal on Computing, vol. 15, no. 3, pp. 310-319, 2003.

[22] B. S. Baker, J. Coffman, and R. . Rivest, "Orthogonal packings in two dimensions," SIAM Journal on Computing, vol. 9, no. 4, pp. 846-855, 1980.

[23] D. Liu and H. Teng, "An improved BL-algorithm for genetic algorithm of the orthogonal packing of rectangles," European Journal of Operational Research, vol. 112, no. 2, pp. 413-420, 1999.

[24] L. H. W. Yeung and W. K. S. Tang, "Strip-packing using hybrid genetic approach," Engineering Applications of Artificial Intelligence, vol. 17, no. 2, pp. 169-177, 2004.

[25] D. Ye, X. Han, and G. Zhang, "A note on online strip packing," Journal of Combinatorial Optimization, vol. 17, no. 4, pp. 417423, 2009.

[26] A. Cassioli and M. Locatelli, "A heuristic approach for packing identical rectangles in convex regions," Computers and Operations Research, vol. 38, no. 9, pp. 1342-1350, 2011.

[27] J. Błazewicz, P. Hawryluk, and R. Walkowiak, "Using a tabu search approach for solving the two-dimensional irregular 
cutting problem," Annals of Operations Research, vol. 41, no. 4, pp. 313-325, 1993.

[28] V. Petridis, S. Kazarlis, and A. Bakirtzis, "Varying fitness functions in genetic algorithm constrained optimization: the cutting stock and unit commitment problems," IEEE Transactions on Systems, Man, and Cybernetics. Part B: Cybernetics, vol. 28, no. 5, pp. 629-640, 1998.

[29] Y. Chen, M. Tang, R. Tong, and J. Dong, "Packing of polygons using genetic simulated annealing algorithm," Journal of Computer-Aided Design and Computer Graphics, vol. 15, no. 5, pp. 598-609, 2003 (Chinese).

[30] R. Andrade and E. G. Birgin, "Symmetry-breaking constraints for packing identical rectangles within polyhedra," Optimization Letters, vol. 7, no. 2, pp. 375-405, 2013.

[31] E. G. Birgin and R. D. Lobato, "Orthogonal packing of identical rectangles within isotropic convex regions," Computers and Industrial Engineering, vol. 59, no. 4, pp. 595-602, 2010.

[32] H.-F. Teng, S. L. Sun, W. H. Ge, and W. X. Zhong, "Layout optimization for the dishes installed on a rotating table-the packing problem with equilibrium behavioural constraints," Science in China A: Mathematics, Physics, Astronomy, vol. 37, no. 10, pp. 1272-1280, 1994.

[33] E. Feng, X. Wang, X. Wang, and H. Teng, "An algorithm of global optimization for solving layout problems," European Journal of Operational Research, vol. 114, no. 2, pp. 430-436, 1999.

[34] Y.-C. Xu, R.-B. Xiao, and M. Amos, "Particle swarm algorithm for weighted rectangle placement," in Proceedings of the $3 \mathrm{rd}$ International Conference on Natural Computation (ICNC '07), China IEEE Press, Haikou, China, August 2007.

[35] Y.-C. Xu, F.-M. Dong, Y. Liu, and R.-B. Xiao, "Genetic algorithm for rectangle layout optimization with equilibrium constraints," Pattern Recognition and Artificial Intelligence, vol. 23, no. 6, pp. 794-801, 2010 (Chinese).

[36] D. H. Wolpert and W. G. Macready, "No free lunch theorems for optimization," IEEE Transactions on Evolutionary Computation, vol. 1, no. 1, pp. 67-82, 1997.

[37] W.-Q. Huang and R.-C. Xu, "Two Quasi-human strategies for the circle packing problem," Science China (Series E), vol. 29, no. 4, pp. 347-353, 1999.

[38] W.-Q. Huang and M. Chen, "Note on: an improved algorithm for the packing of unequal circles within a larger containing circle," Computers and Industrial Engineering, vol. 50, no. 3, pp. 338-344, 2006.

[39] W.-Q. Huang and T. Ye, "Quasi-physical algorithm for the equal circle packing problem," Journal System Science \& Math, vol. 50, no. 3, pp. 993-1001, 2008.

[40] W.-Q. Huang and T. Ye, "Quasi-physical global optimization method for solving the equal circle packing problem," Science China: Information Sciences, vol. 54, no. 7, pp. 1333-1339, 2011.

[41] Y. G. Stoyan and G. N. Yaskov, "Mathematical model and solution method of optimization problem of placement of rectangles and circles taking into account special constraints," International Transactions in Operational Research, vol. 5, no. 1, pp. 45-57, 1998.

[42] H.-F. Teng, Z.-Q. Li, Y.-J. Shi, and Y.-S. Wang, "An approach to constructing isomorphic or non-isomorphic layout pattern," Chinese Journal of Computers, vol. 29, no. 6, pp. 985-991, 2006.

[43] H.-F. Teng, Y. Chen, W. Zeng, Y.-J. Shi, and Q.-H. Hu, "A dualsystem variable-grain cooperative coevolutionary algorithm: satellite-module layout design," IEEE Transactions on Evolutionary Computation, vol. 14, no. 3, pp. 438-455, 2010.
[44] E.-M. Feng, Z.-H. Gong, C.-Y. Liu, and Z. Xu, "Improved GA for satellite module layout problem with performance constraints," Journal of Dalian University of Technology, vol. 45, no. 3, pp. 457463, 2005. 


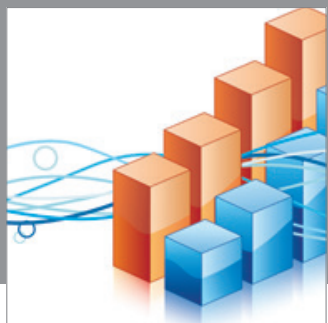

Advances in

Operations Research

mansans

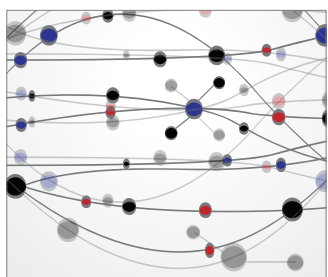

The Scientific World Journal
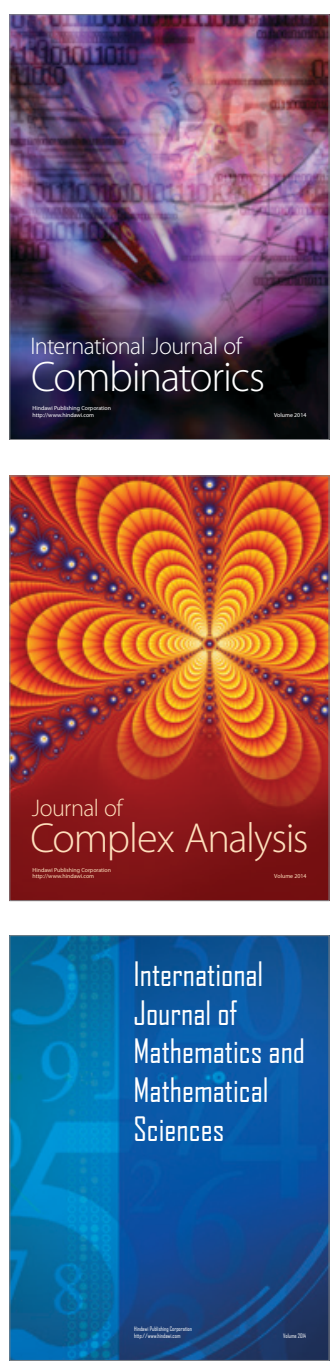
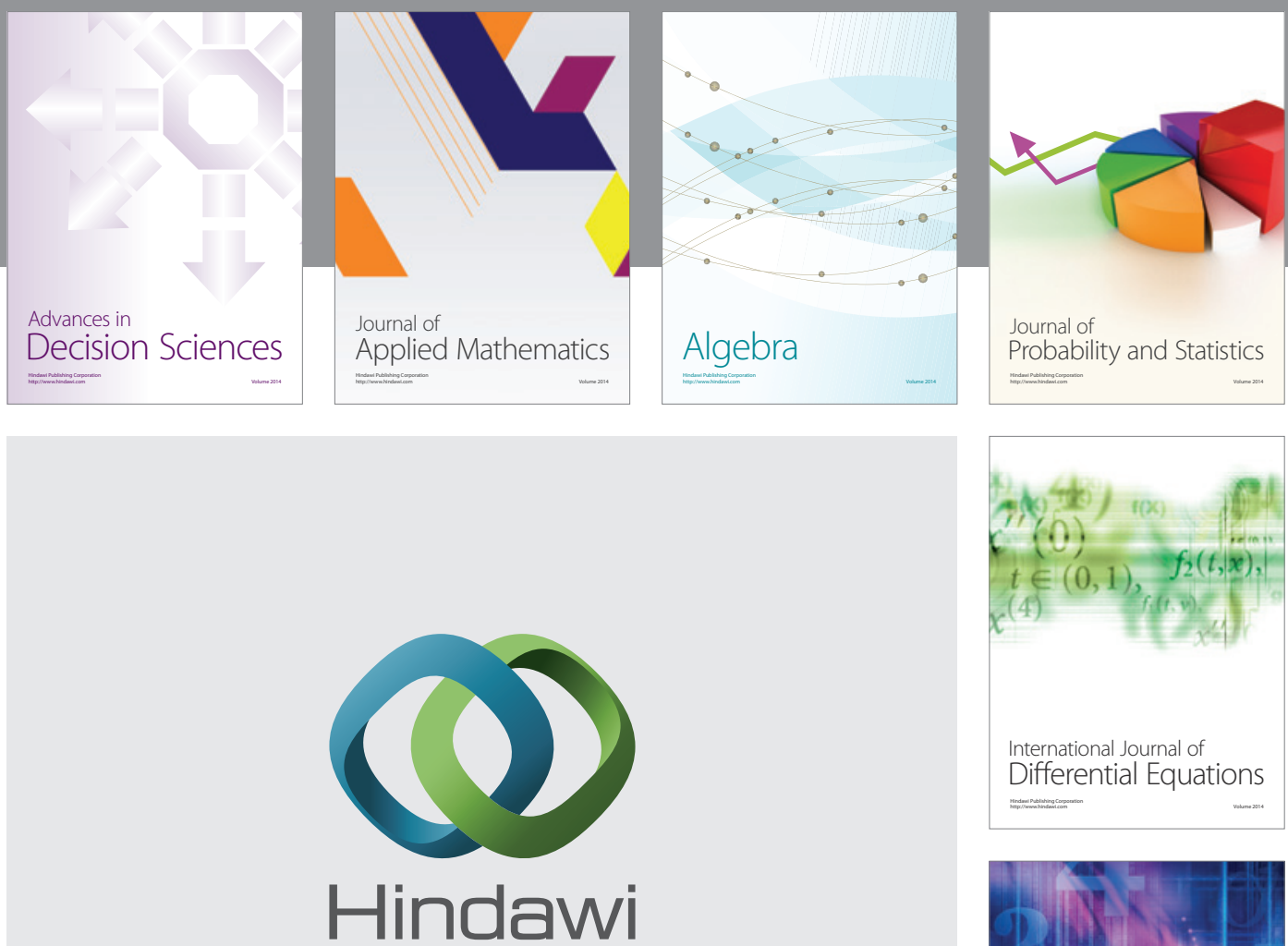

Submit your manuscripts at http://www.hindawi.com
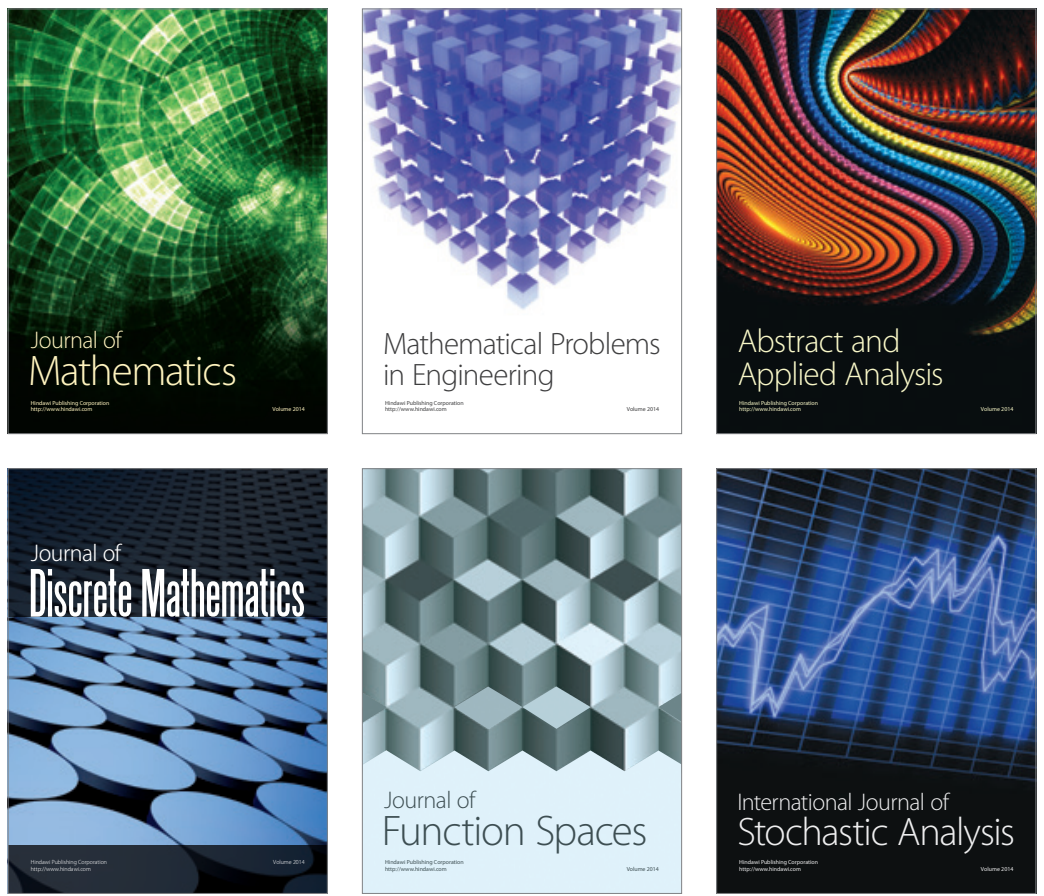

Journal of

Function Spaces

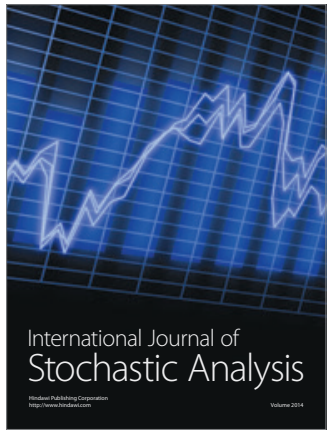

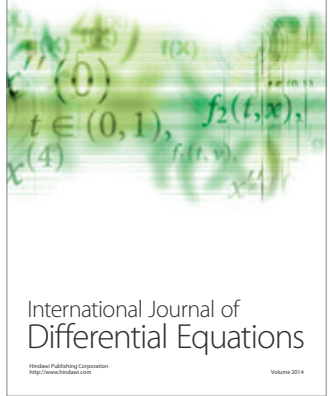
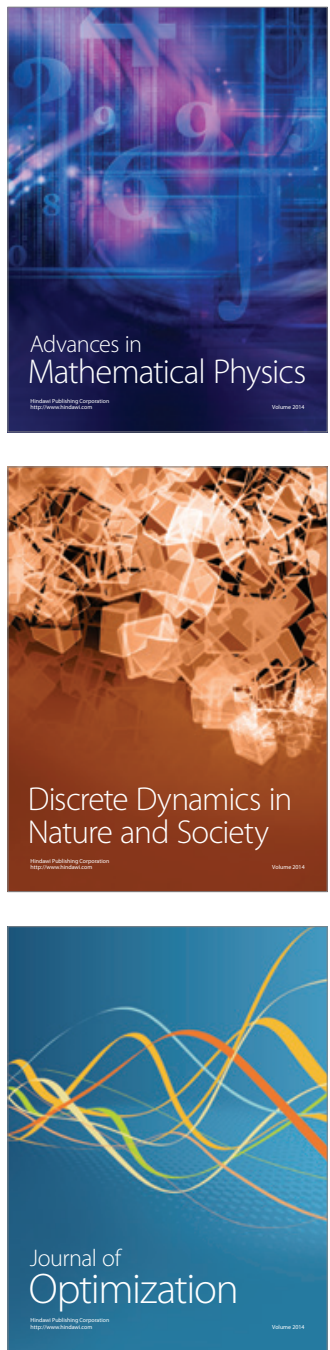